\title{
Phase-Amplitude Dynamics in terms of Extended Response Functions: Invariant Curves and Arnold Tongues
}

\author{
Oriol Castejón ${ }^{1}$ and Antoni Guillamon ${ }^{1,2}$ \\ ${ }^{1}$ Departament de Matemàtiques (Universitat Politècnica de Catalunya), Av. Dr. Marañón 44-50, 08028 Barcelona \\ ${ }^{2}$ Barcelona Graduate School of Mathematics BGSMath*
}

(Dated: September 26, 2019)

Phase response curves (PRCs) have been extensively used to control the phase of oscillators under perturbations. Their main advantage is the reduction of the whole model dynamics to a single variable (phase) dynamics. However, in some adverse situations (strong inputs, high-frequency stimuli, weak convergence,...), the phase reduction does not provide enough information and, therefore, PRC lose predictive power. To overcome this shortcoming, in the last decade, new contributions have appeared that allow to reduce the system dynamics to the phase plus some transversal variable that controls the deviations from the asymptotic behaviour. We call this setting extended response functions. In particular, we single out the phase response function (PRF, a generalization of the PRC) and the amplitude response function (ARF) that account for the above-mentioned deviations from the oscillating attractor. It has been shown that in adverse situations, the PRC misestimate the phase dynamics whereas the PRF-ARF system provides accurate enough predictions. In this paper, we address the problem of studying the dynamics of the PRF-ARF systems under periodic pulsatile stimuli. This paradigm leads to a two-dimensional discrete dynamical system that we call $2 \mathrm{D}$ entrainment map. By using advanced methods to study invariant manifolds and the dynamics inside them, we construct an analytico-numerical method to track the invariant curves induced by the stimulus as two crucial parameters of the system increase (the strength of the input and its frequency). Our methodology also incorporates the computation of Arnold tongues associated to the $2 \mathrm{D}$ entrainment map. We apply the method developed to study inner dynamics of the invariant curves of a canonical type II oscillator model. We further compare the Arnold tongues of the 2D map with those obtained with the map induced only by the PRC, which give already noticeable differences. We also observe (via simulations) how high-frequency or strong enough stimuli break up the oscillatory dynamics and lead to phase-locking, which is well captured by the $2 \mathrm{D}$ entrainment map.

Keywords: Phase-amplitude response curves; Invariant manifolds; Entrainment maps; type II oscillators

\footnotetext{
* Corresponding author: A. Guillamon (antoni.guillamon@upc.edu)
} 


\section{Introduction}

Phase response curves (PRCs) constitute a systematic tool to understand synchronisation between oscillators or their entrainment to external stimuli. The purpose of this theory is predicting the change of phase elicited by a given perturbation in terms of the phase of the unperturbed oscillatory system, which we assume to have an attracting limit cycle. In single neuron models, each complete oscillation typically corresponds to a complete action potential and the goal of phase response theory is giving a prediction of the delay or advancement of the first spike after the perturbation, which can come either from synaptic inputs or from direct stimulation. Most of the literature on phase response curves has been developed in relation to neuroscience (see [1-3]), but the concept transcends to any other field in which oscillations are present.

On the other hand, most theoretical contributions are devoted to the case in which the oscillator is assumed to remain on its asymptotic state, a constraint generally known as phase reduction, which will be referred also as asymptotic-state hypothesis in the context of this paper. The asymptotic-state hypothesis is widely valid but it fails in transient states, generally because of strong, highly repetitive or noisy stimuli, or due to the slow convergence to the asymptotic oscillatory state, that is, the attracting limit cycle. In order to go beyond the limits of the phase reduction, recent literature has focused on the control of the phase response out of the limit cycle (see [4-9]), a problem that is intertwined with the progress about the computation of the so-called isochrons associated to the limit cycle (see [4,10-13]). It is worth mentioning that under the asymptotic-state hypothesis, the PRC is a function that maps the phase $\theta$ of the oscillator onto the phase change elicited by a given stimulus $I_{\text {stim }}$. However, when the PRC concept is extended to a neighbourhood of the limit cycle (see [4]), one has to consider a $(n-1)$-dimensional amplitude variable transversal to the limit cycle. Assuming that there is a privileged direction (corresponding to the eigenvalue of the Poincaré map closest to the unit circle, see $[8,9,14])$, that will control at first order the proximity to the limit cycle (technical details explained below), then the PRC naturally extends to a system of two variables, namely the phase and this privileged amplitude variable (see Figure 1), and two response functions, named phase and amplitude response functions (PRF and ARF, respectively), see [5] and Section II.

Our goals are motivated by the open question of studying the influence of stimuli on the dynamics of oscillators, which in the context of PRCs has been addressed, for instance, in [15-17]. In order to quantify the validity of the asymptotic-state hypothesis, in [5] we studied the differences in predicting the entrainment to a periodic pulse stimulus, which induces an entrainment map; more specifically, a 1D discrete dynamical system that maps the value of the state variables before a stimulus to the value before the next one, see 


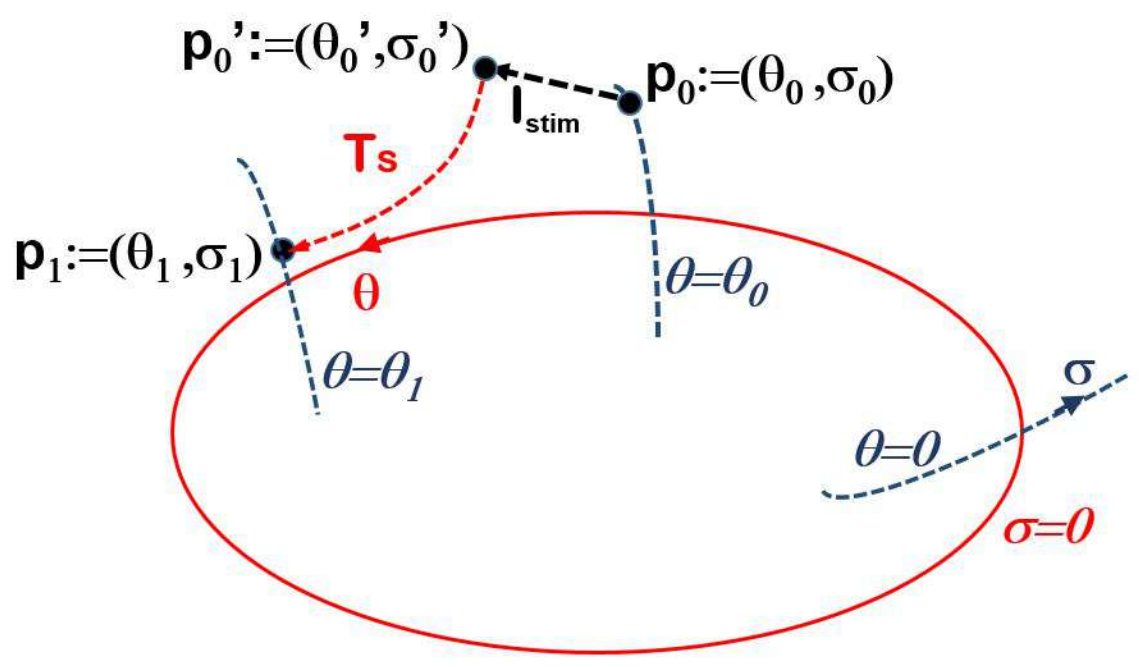

Figure 1: Schematic representation of the phase-amplitude map induced by a periodic pulse train.

The neighbourhood of the limit cycle can be parameterized by the phase $(\theta)$ and amplitude $(\sigma)$ variables, the limit cycle corresponding to $\sigma=0$ (solid line, in red). Several isochrons (dashed curves transversal to the limit cycle, in blue) are shown; we usually choose the isochron containing the intersection between the first nullcline and the limit cycle to be the 0 -isochron $(\theta=0)$. The $2 \mathrm{D}$ map sends $p_{0}:=\left(\theta_{0}, \sigma_{0}\right)$ to $\left(\theta_{1}, \sigma_{1}\right)$ as follows: at $p_{0}$ the system receives a pulse input $I_{\text {stim }}$ which determines the kick to $p_{0}^{\prime}:=\left(\theta_{0}^{\prime}, \sigma_{0}^{\prime}\right)$; then, we integrate (dashed curve, in red) along the flow $T_{s}$ time units (stimulation period) landing on $p_{1}$. The phase reduction approach assumes that $\sigma$ is always zero.

Figure 1. Entrainment maps are a useful tool to make predictions about biological rhythms (see for instance $[18,19])$. Note that, under the asymptotic-state hypothesis, this paradigm induces a $1 \mathrm{D}$ entrainment map in the phase space, whereas in the extended version, it induces a $2 \mathrm{D}$ entrainment map in the phase-amplitude space.

In [5], we compared the differences between the 1D and the 2D approaches in terms of the hyperbolicity of the limit cycle and the tilt of its isochrons, see also [6]. We focused our analysis on the behavior of the rotation numbers associated to the phase variable of both entrainment mapsand detected important differences for the two cases as we increased the input strength $\left(\left\|I_{\text {stim }}\right\|\right.$ in Figure 1) or the stimulation frequency $\left(1 / T_{s}\right.$ in Figure 1). In a minimal model allowing for an analytical computation of the isochrons and all class of response curves, we showed that the rotation number for the $1 \mathrm{D}$ entrainment map could have absolute errors (compared to the exact rotation number) two orders of magnitude higher than the 
2D entrainment. In the cases we studied, the error for the $1 \mathrm{D}$ entrainment map could be of order $10^{-2}$, which implies dramatic misestimations of the perturbed phase under a high-frequency input (for instance, a bursting-like one or a synchronized bombardment from a pool of presynpatic neurons).

The rotation numbers obtained in [5] were referred to the phase defined by the limit cycle, no matter the perturbation created a new periodic attractor with a new phase or even broke up the periodic dynamics, but the dynamics of discrete dynamical systems generated by these entrainment maps and their limitations in predicting the real behaviour of the phase and amplitude variables have not been analyzed in depth. In this paper, we perform this analysis with the aim of understanding both the dynamics of phase-amplitude variables when the system is subjected to periodic pulsatile inputs and the undergoing bifurcations as we increase either the strength or the frequency of the perturbation. In particular, we are interested in checking the existence of the invariant curves of the $2 \mathrm{D}$ map, understanding the dynamics inside these invariant curves and explaining the bifurcations that lead to changes in this internal dynamics or to a transition from the invariant curve attractor regime to a fixed-point attractor regime. Moreover, we aim to describe how the periods of the dynamics inside the invariant curves of the 2D map are organized within the stimulus strength-frequency parameter space.

As far as we know, this is the first time that the intrinsic dynamics of these 2D entrainment maps is studied. Therefore, we focus on a canonical form of type II oscillators that enables us to better dissect the basic involved mechanisms. The model and the two corresponding entrainment maps are presented in Section II, together with a brief update of the results from [5], for the sake of self-containment. By studying this canonical example we aim at better distinguishing the main differences between the 1D and the 2D corresponding discrete dynamical systems, understanding more deeply the dynamics of the 2D map and obtaining good predictions of their long-term behavior. Moreover, we aim at giving an insight of what can happen in more complex and realistic models in neuroscience, for which this analytico-numerical study that we carry on here would be more cumbersome and perhaps less illustrative.

In order to tackle the above-mentioned goals, we take advantage of well-known methods from the theory of discrete dynamical systems and we propose two different numerical schemes. Since our 2D entrainment map is similar in many aspects to the Arnold family of annulus diffeomorphisms studied in [20], we adapt their techniques to compute the invariant curves and their internal dynamics, and to localize the parameter regions corresponding to specific periods of this internal dynamics via Arnold tongues. These techniques, see Section III, are based on Taylor expansions of the invariant curves and their internal dynamics. The results that we present are obtained using this methodology, but we also propose another approach to com- 
pute the invariant curves, namely a Newton-like method (see [21]) consisting of solving the invariance equation derived from the parameterization method (see [22]). For the sake of completeness, we keep the development of this method in Appendix A. The results obtained with these two methods are exposed in Section IV and further discussed in Section V.

\section{Background and model}

In this section, we present the system that we will perturb with a periodic pulse stimulus. As mentioned in the Introduction, we focus on a canonical form of type II oscillators, with two relevant parameters: the degree of hiperbolicity and the tilt of the isochrons. The model was chosen to be simple to allows more insight into the entrainment mechanisms that we want to study. In Section II A, we review the definition of phase and amplitude response functions and apply it to obtain the corresponding exact expressions (PRF and ARF, respectively) for this simple model. These response functions constitute the main ingredients to build up the entrainment maps (Section II B) induced by the perturbation which, of course, will involve the frequency and the strength of the periodic pulse stimulus.

\section{A. A simple canonical model}

We choose a simple canonical model having a limit cycle and endowed with two parameters, $\alpha$ and $a$, controlling, respectively, the hyperbolicity of the limit cycle (that is, its attractiveness) and the isochronlimit cycle relative position (also referred to as the tilt of the isochrons). In polar coordinates, the vector field writes as

$$
\mathcal{X}:=\left\{\begin{array}{l}
\dot{r}=\alpha r\left(1-r^{2}\right), \\
\dot{\varphi}=1+\alpha a r^{2}
\end{array}\right.
$$

with $a \geq 0$ and $\alpha>0$. In Cartesian coordinates, system (1) writes as

$$
X:=\left\{\begin{array}{l}
\dot{x}=\alpha x\left(1-\left(x^{2}+y^{2}\right)\right)-y\left(1+\alpha a\left(x^{2}+y^{2}\right)\right), \\
\dot{y}=\alpha y\left(1-\left(x^{2}+y^{2}\right)\right)+x\left(1+\alpha a\left(x^{2}+y^{2}\right)\right) .
\end{array}\right.
$$

The circle $r=1$ is a limit cycle of (1), that will be called $\Gamma$. The dynamics on $\Gamma$ is given by $\dot{\varphi}=$ $1+\alpha a$. Therefore, $\varphi(t)=\varphi_{0}+(1+\alpha a) t \bmod 2 \pi$ and the period of the limit cycle is $T_{0}=2 \pi /(1+$ $\alpha a)$. A parameterization of the limit cycle in terms of the phase $\theta=t / T_{0}$, for $\theta \in[0,1)$ is $\gamma(\theta)=$ 
$(\cos (2 \pi \theta), \sin (2 \pi \theta))$. The characteristic exponent of $\Gamma$ is $\lambda=-2 \alpha T_{0}=-4 \alpha \pi /(1+\alpha a)$. Hence, the larger is $\alpha$, the stronger will be the attraction to the limit cycle.

In [5], we provided the explicit expression of both the isochrons associated to $\Gamma$ and the expression of the phase-amplitude response curves. For the sake of completeness, we briefly explain how these results are obtained. First of all, note that the change of coordinates $(x, y)=K(\theta, \sigma)$ with

$$
K(\theta, \sigma)=\left(\sqrt{\frac{1}{1-2 \alpha \sigma}} \cos \left(2 \pi \theta+\frac{1}{2} a \ln (1-2 \alpha \sigma)\right), \sqrt{\frac{1}{1-2 \alpha \sigma}} \sin \left(2 \pi \theta+\frac{1}{2} a \ln (1-2 \alpha \sigma)\right)\right),
$$

where $\theta \in[0,1)$ and $\sigma<(2 \alpha)^{-1}$, brings the system (2) into

$$
\begin{aligned}
& \dot{\theta}=1 / T_{0}, \\
& \dot{\sigma}=\lambda \sigma / T_{0}=-2 \alpha \sigma .
\end{aligned}
$$

This is the basic transformation within the parameterization method, see [4], and gives valuable information about the phase-amplitude dynamics.

Indeed, notice that the function $K$ can be easily inverted using that $r^{2}=x^{2}+y^{2}=(1-2 \alpha \sigma)^{-1}$ and $\arctan \left(\frac{y}{x}\right)=2 \pi \theta+\frac{1}{2} a \ln (1-2 \alpha \sigma)$, leading to $K^{-1}(x, y)=:(\Theta(x, y), \Sigma(x, y))$, where

$$
\Theta(x, y)=\frac{1}{2 \pi}\left(\arctan \left(\frac{y}{x}\right)-\frac{1}{2} a \ln \left(\frac{1}{r^{2}}\right)\right), \quad \Sigma(x, y)=\frac{1}{2 \alpha}\left(1-\frac{1}{r^{2}}\right) .
$$

The functions $K, \Theta$ and $\Sigma$ are very relevant to control the change in phase and amplitude, respectively, produced by a brief stimulus. On one side, for a fixed $\theta_{0}=\in[0,1)$, the curve defined by $K\left(\theta_{0}, \sigma\right)$ (equivalently, $\left.\Theta=\theta_{0}\right)$ corresponds to the $\theta_{0}$-isochron; on the other side, the curve defined by $K\left(\theta, \sigma_{0}\right)$ for a fixed $\sigma_{0}=\in\left(-\infty, 1 /(2 \alpha)\right.$ ) (equivalently, $\left.\Sigma=\sigma_{0}\right)$ corresponds to the so-called $\sigma_{0}$-isostable. Moreover, the phase and amplitude response functions can be defined directly from these functions as the corresponding dot product with the stimulus vector (see [5]):

$$
\operatorname{PRF}\left(x, y ; I_{\text {stim }}\right)=\nabla \Theta(x, y) \cdot I_{\text {stim }}, \quad \operatorname{ARF}\left(x, y ; I_{\text {stim }}\right)=\nabla \Sigma(x, y) \cdot I_{\text {stim }},
$$

where $\nabla$ stands for the gradient.

In our example, we have that $\nabla \Theta(x, y)=\frac{1}{2 \pi r^{2}}(-y+a x, x+a y)$, and $\nabla \Sigma(x, y)=\left(\frac{x}{\alpha r^{4}}, \frac{y}{\alpha r^{4}}\right)$.

Without loss of generality, due to the symmetry of the problem, we take $I_{\text {stim }}=(1,0)$. Therefore, the phase and amplitude response functions can be written in the $(\theta, \sigma)$ variables as

$$
\operatorname{PRF}(K(\theta, \sigma))=-\frac{\sqrt{1-2 \alpha \sigma}}{2 \pi}\left(\sin \left(2 \pi \theta+\frac{1}{2} a \ln (1-2 \alpha \sigma)\right)-a \cos \left(2 \pi \theta+\frac{1}{2} a \ln (1-2 \alpha \sigma)\right)\right)
$$


and

$$
A R F(K(\theta, \sigma))=\frac{(1-2 \alpha \sigma)^{3 / 2}}{\alpha} \cos \left(2 \pi \theta+\frac{1}{2} a \ln (1-2 \alpha \sigma)\right) .
$$

We remark that, given a point $\gamma\left(\theta_{0}\right) \in \Gamma$, the angle $(\beta)$ between the isochron $\Theta=\theta_{0}$ and the limit cycle at this point is independent of $\theta_{0}$ (by symmetry) and

$$
\cos \beta=\frac{\gamma^{\prime}\left(\theta_{0}\right) \cdot \nabla \Theta^{\perp}\left(\gamma\left(\theta_{0}\right)\right)}{\left\|\gamma^{\prime}\left(\theta_{0}\right)\right\|\left\|\nabla \Theta^{\perp}\left(\gamma\left(\theta_{0}\right)\right)\right\|}=\frac{a}{\sqrt{1+a^{2}}} .
$$

Observe that for $a=0$, the isochrons will be orthogonal to the limit cycle, and they will become tangent to it as $a$ goes to infinity (see Figure 2).

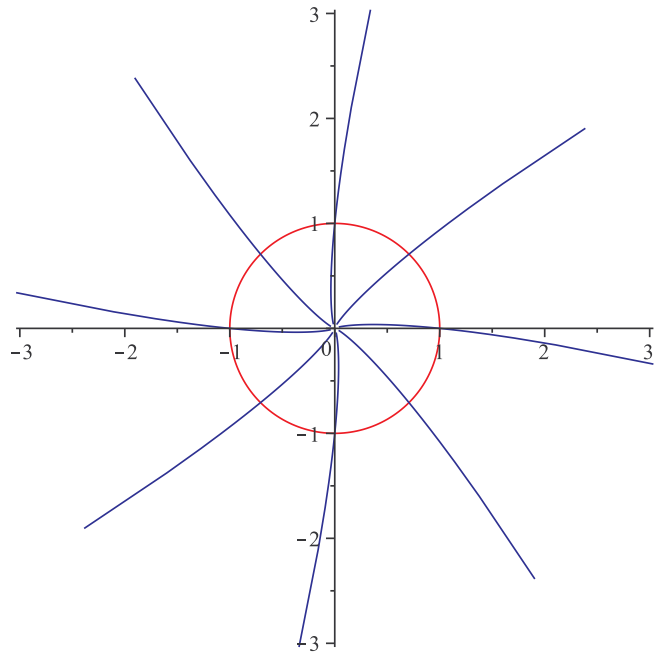

(a) $a=0.1$

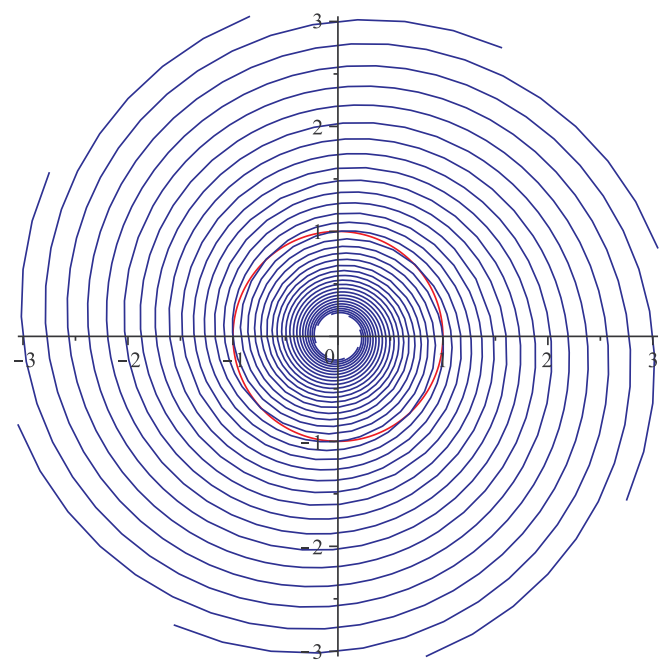

(b) $a=10$

Figure 2: Influence of parameter $a$ on the tilt of isochrons. The limit cycle (red) of system (1) and some isochrons (blue) for different values of the parameter $a$. In both cases, $\alpha=10$. For small values of $a$ (left panel), the isochrons are almost radial, whereas large values of $a$ (right panel) produce a significative tilt of the isochrons which become almost tangent to the limit cycle, thus increasing the phase sensitivity under perturbations.

\section{B. Entrainment maps}

We will force our system with pulse-trains of period $T_{s} \ll T_{0}$, nearby a limit cycle $\Gamma$ of period $T_{0}$ and characteristic exponent $\lambda$. More specifically, we consider a generic oscillator, and assume that it is perturbed 
with an external instantaneous stimulus of amplitude $\varepsilon$ in the voltage direction every $T_{s}$ time units, that is:

$$
\dot{\mathbf{x}}=X(\mathbf{x})+\varepsilon v \sum_{j=0}^{N} \delta\left(t-j T_{s}\right),
$$

where $v=(1,0)$ and $\delta$ is the Dirac delta function. This system can represent, for example, a neuron receiving an idealized synaptic input from other neurons.

Remark II.1 In the sequel, we will also use $\omega_{s}=1 / T_{s}$, the frequency of the stimulus, and $\omega_{0}=1 / T_{0}$, the frequency of the limit cycle $\Gamma$. Then, the quotient $\omega_{s} / \omega_{0}$ indicates how many inputs receives the oscillator in one period. In the context of neuroscience, the paradigm that we analyze in this paper may correspond to different situations. For instance, the quotient $\omega_{s} / \omega_{0}$ could represent the number of presynaptic neurons to the target neuron, assuming that all presynaptic neurons fire asynchronously at the same firing rate, close to $\omega_{0}$, and $\varepsilon$ is the height of a postsynaptic potential. Still assuming the same firing rate for all the neurons in the population, increasing synchrony would imply a decrease of the quotient $\omega_{s} / \omega_{0}$ together with an increase in $\varepsilon$; it could still fall into our idealized framework although any source heterogeneity may break it. We will return to this question in the discussion, Section V.

We shall just focus on the maps given in (5) and (6) obtained from the canonical model (1) under the pulseperiodic stimuli of frequency $\omega_{s}$. Our purpose in studying this particular example is to understand more deeply the 2D map, to establish more precisely the main differences between the 1D and the 2D approaches, and to make better predictions of their long-term behavior. This is a minimal model, so that we expect that this study gives insight of what can happen in more complex and realistic models in neuroscience, for which this numerical study that we shall carry on would be more cumbersome and perhaps less illustrative.

In order to know the evolution of this perturbed oscillator after each time period $T_{s}$, it is enough to know how the variables $\theta$ and $\sigma$ change. We recall that the variation of the variable $\theta$ produced by an external stimulus $I_{\text {stim }}:=(\varepsilon, 0)$ is given, in first order of the stimulus strength $\varepsilon$, by the PRF obtained in (5). Similarly, the variation of the variable $\sigma$ is given in first order by the ARF obtained in (6). Hence, we can consider the following map, which approximates the position of the oscillator at the moment preceding next kick:

$$
\begin{aligned}
\theta_{n+1} & =\theta_{n}+\varepsilon \operatorname{PRF}\left(\theta_{n}, \sigma_{n}\right)+\frac{T_{s}}{T_{0}} \quad(\bmod 1), \\
\sigma_{n+1} & =\left(\sigma_{n}+\varepsilon \operatorname{ARF}\left(\theta_{n}, \sigma_{n}\right)\right) e^{\lambda T_{s} / T_{0}} .
\end{aligned}
$$

Notice that, on one hand, the three terms of the right-hand side of the first equation account for, respectively, (1) the phase at step $n,(2)$ the change in phase produced by the kick at step $n$ (governed by the PRF) and 
(3) the phase elapsed between the $n$-th and the $(n+1)$-th kicks. On the other hand, the right-hand side of the second equation account for the amplitude at step $n$ plus the change in amplitude produced by the kick at step $n$ (governed by the ARF), altogether multiplied by $e^{\lambda T_{s} / T_{0}}$, which reflects the variation of amplitude due to the flow of $X$, see (4). See Figure 1 for a schematic representation of the elements involved in the map (8).

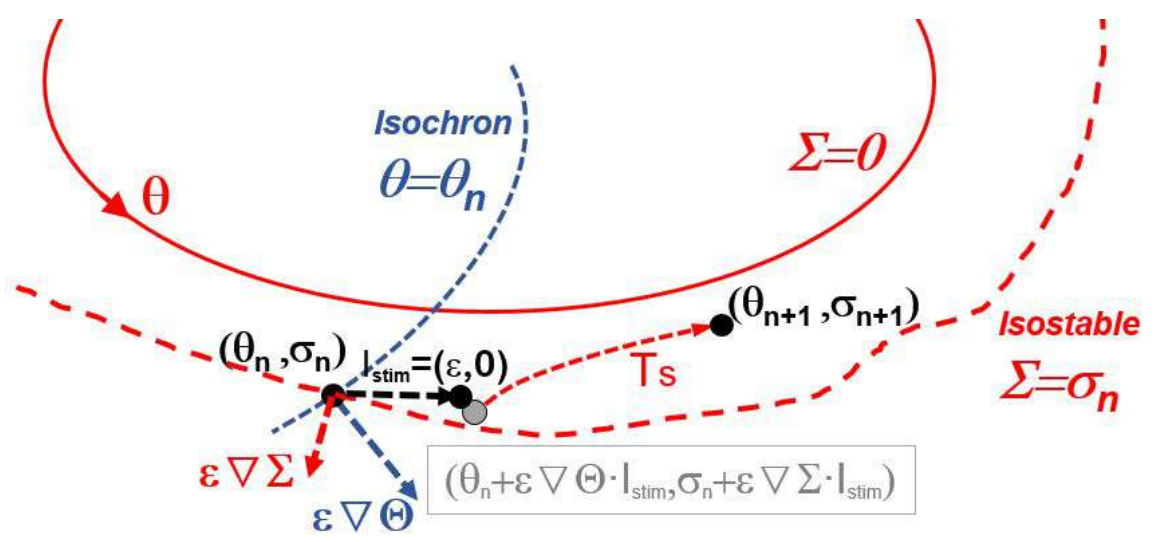

Figure 3: Schematic representation of the elements involved in the map (8). We apply a pulse stimulus $I_{\text {stim }}=(\varepsilon, 0)$ at the point $\left(\theta_{n}, \sigma n\right)$. This perturbation alters the current state of the system from $\left(\theta_{n}, \sigma n\right)$ to the point in black at the tip of the arrow of $I_{\text {stim }}$. The PRF and ARF give an approximation of this point by means of the expression $\left(\theta_{n}+\varepsilon P R F\left(\theta_{n}, \sigma_{n}\right), \sigma_{n}+\varepsilon A R F\left(\theta_{n}, \sigma_{n}\right)\right)$ (grey point). To reach $\left(\theta_{n+1}, \sigma n+1\right)$, we complete the action of map 8 by integrating (4) $T_{s}$ time units, which is equivalent to add $T_{s} / T_{0}$ to the phase and multiply by $\exp \left(\lambda T_{s} / T_{0}\right)$ the amplitude.

We will compare the map (8) with the map obtained by considering the classical PRC (see, for instance, [1, Ch. 10]), which is:

$$
\theta_{n+1}=\theta_{n}+\varepsilon \operatorname{PRC}\left(\theta_{n}\right)+\frac{T_{s}}{T_{0}} \quad(\bmod 1)
$$

Maps (9) and (8) are, respectively, the 1D and 2D entrainment maps that we mention in the Introduction. In the $1 \mathrm{D}$ case we are assuming that the perturbation happens always on the limit cycle, and therefore $\sigma_{n}=0$ for all $n$. In [5], we explored the limits of validity of the latter hypothesis in terms of $\omega_{s}$ and $\varepsilon$. Here, we want to study the dynamics of these two entrainment maps. Since the 1D entrainment map is a particular 
case, we will develop the methodology for the $2 \mathrm{D}$ entrainment and then use it in both cases for the sake of comparison. We shall denote by $F_{\varepsilon, \omega}: \mathbb{T} \times \mathbb{R} \rightarrow \mathbb{T} \times \mathbb{R}$ the $2 \mathrm{D}$ map associated to the discrete dynamical system (8):

$$
\begin{aligned}
F_{\varepsilon, \omega}: \mathbb{T} \times \mathbb{R} & \longrightarrow \mathbb{T} \times \mathbb{R} \\
\left(\begin{array}{l}
\theta \\
\sigma
\end{array}\right) & \longmapsto\left(\begin{array}{cc}
\theta+\omega+\varepsilon \operatorname{PRF}(\theta, \sigma) & (\bmod 1) \\
(\sigma+\varepsilon A R F(\theta, \sigma)) e^{\lambda \omega} &
\end{array}\right),
\end{aligned}
$$

where $\lambda<0$ and $\omega:=\omega_{s}=T_{s} / T_{0}$ is the ratio between the stimulation period and the period of the underlying limit cycle of the unperturbed system.

\section{Computation of invariant curves and Arnold tongues using Taylor expansions}

In this section we implement a method to compute the invariant curves and the dynamics inside them. For the theoretical background of this section we follow the ideas found in [20]. The implementation of the numerical methods is based on [23] and [24] (see also [25]). For the whole procedure, we use automatic differentiation tools which, in particular, facilitate the computation of the so-called Arnold tongues.

\section{A. The invariance equations}

Let us fix $\omega=p / q \in \mathbb{Q}$. To find the invariant curve of the map $F_{\varepsilon, \omega}$ defined in (10) and the dynamics inside it, we look for invariant curves of the form $\sigma=g(\theta, \varepsilon)$ and denote the dynamics inside these invariant curves by $h(\theta, \varepsilon)$. Both $g$ and $h$ are defined implicitly from (10) and they must satisfy the following invariance equations:

$$
h(\theta, \varepsilon)=\theta+\omega+\varepsilon \operatorname{PRF}(\theta, g(\theta, \varepsilon)),
$$

and

$$
g(h(\theta, \varepsilon))=(g(\theta, \varepsilon)+\varepsilon A R F(\theta, g(\theta, \varepsilon))) e^{\lambda \omega} .
$$

Expanding $h$ and $g$ in orders of $\varepsilon$, that is,

$$
h(\theta, \varepsilon)=\sum_{n \geq 0} h_{n}(\theta) \varepsilon^{n}, \quad g(\theta, \varepsilon)=\sum_{n \geq 0} g_{n}(\theta) \varepsilon^{n},
$$

we can use equations (11) and (12) to compute the $n$-th order functions $h_{n}(\theta)$ and $g_{n}(\theta)$, for $n=0, \ldots, N$. 
For $\varepsilon=0$, there is no perturbation and so the limit cycle of system (1) is indeed the invariant curve of $F_{0, \omega}$. Moreover, since the phase is normalized, we can interpret the dynamics on this invariant curve as being a pure rotation with rotation number $p / q$. For a fixed $\varepsilon>0$ small enough, this $p / q$-rotation generically persists for an $\omega$-interval whose width depends on $\varepsilon$. We say that $(\omega, \varepsilon) \in T_{p, q}$ if and only if there exist $\left(\theta^{*}, \sigma^{*}\right) \in \mathbb{T} \times \mathbb{R}$ such that $F_{\varepsilon, \omega}^{q}\left(\theta^{*}, \sigma^{*}\right)=\left(\theta^{*}+2 \pi p, \sigma^{*}\right)$. We call $T_{p, q}$ the Arnold tongue of rotation number $p / q$. The boundaries of the Arnold tongues correspond to saddle-node bifurcation points of the function $F_{\varepsilon, \omega}^{q}(\theta, \sigma)$, and they can be parameterized in the plane $(\omega, \varepsilon)$ by two curves $\omega_{ \pm}(\varepsilon)$ such that $\omega(0)=p / q$. We will use the notation $\omega_{ \pm}(\varepsilon)=p / q+\delta_{ \pm}(\varepsilon)$, where $\delta(0)=0$ to denote the two boundaries of the $p / q$-Arnold tongue. With this notation, the invariance equations (11) and (12) write as

$$
h(\theta, \varepsilon, \delta)=\theta+\omega+\delta+\varepsilon \operatorname{PRF}(\theta, g(\theta, \varepsilon, \delta)),
$$

and

$$
g(h(\theta, \varepsilon, \delta), \varepsilon, \delta)=(g(\theta, \varepsilon, \delta)+\varepsilon A R F(\theta, g(\theta, \varepsilon, \delta))) e^{\lambda(\omega+\delta)},
$$

where $\omega=p / q$ is fixed, and $h$ and $g$ expand in orders of $\varepsilon$ and $\delta$ as

$$
h(\theta, \varepsilon, \delta)=\sum_{j, k \geq 0} h_{j k}(\theta) \varepsilon^{j} \delta^{k}, \quad g(\theta, \varepsilon, \delta)=\sum_{j, k \geq 0} g_{j k}(\theta) \varepsilon^{j} \delta^{k}
$$

Substituting them in (13) and (14), we obtain the following computational scheme:

$$
\sum_{j, k \geq 0} h_{j k}(\theta) \varepsilon^{j} \delta^{k}=\theta+\omega+\delta+\varepsilon \operatorname{PRF}\left(\theta, \sum_{j, k \geq 0} g_{j k}(\theta) \varepsilon^{j} \delta^{k}\right),
$$

and

$$
\begin{aligned}
& \sum_{j, k \geq 0} g_{j k}\left(\sum_{m, n \geq 0} h_{m n}(\theta) \varepsilon^{m} \delta^{n}\right) \varepsilon^{j} \delta^{k} \\
= & \left(\sum_{j, k \geq 0} g_{j k}(\theta) \varepsilon^{j} \delta^{k}+\varepsilon A R F\left(\theta, \sum_{j, k \geq 0} g_{j k}(\theta) \varepsilon^{j} \delta^{k}\right)\right) e^{\lambda(\omega+\delta)} .
\end{aligned}
$$

Equations (15) and (16) are solved numerically order by order using automatic differentiation tools, specially for the PRF and ARF functions which are combinations of trigonometric and logarithmic functions, see equations (5) and (6). In particular, we first solve the term of order zero of both equations analytically, which can be done easily. After that, we also solve analytically the terms independent of $\varepsilon$. Finally, the 
higher-order terms can be solved numerically once the previous ones are known, so that one just needs to proceed inductively to obtain the subsequent orders. We shall now explain this procedure in more detail.

Concerning the independent terms of both invariance equations, equating the terms that are independent of $\varepsilon$ and $\delta$ in both sides of equation (15) we readily obtain

$$
h_{00}(\theta)=\theta+\omega
$$

Proceeding in a similar way for equation (16) and taking into account that $h_{00}(\theta)=\theta+\omega$, we obtain

$$
g_{00}(\theta+\omega)=g_{00}(\theta) e^{\lambda \omega}
$$

Equation (17) can be solved expanding both sides in Fourier series and equating the Fourier coefficients. More precisely, if we write

$$
g_{00}(\theta)=\sum_{l \in \mathbb{Z}} g_{00}^{l} e^{2 \pi i l \theta}
$$

then equation (17) yields the following equation for each $l \in \mathbb{Z}$ :

$$
g_{00}^{l}\left(e^{2 \pi i l \omega}-e^{\lambda \omega}\right)=0
$$

Since $\lambda \in \mathbb{R} \backslash\{0\}$, clearly $e^{2 \pi i l \omega}-e^{\lambda \omega} \neq 0$ for all $l \in \mathbb{Z}$, so that one obtains straightforwardly:

$$
g_{00}^{l}=0 \quad \text { for all } l \in \mathbb{Z}, \quad g_{00}(\theta)=0
$$

We now focus on the terms $h_{0 k}$ and $g_{0 k}$ with $k \geq 1$. From equation (15) it is straightforward to see that

$$
h_{01}(\theta) \equiv 1, \text { and } h_{0 k}(\theta) \equiv 0, \text { for } k \geq 2 .
$$

In order to compute $g_{0 k}(\theta)$, we proceed in a different way. We first observe that from the previous computations we know that $h(\theta, 0, \delta)=\theta+\omega+\delta$. Hence, setting $\varepsilon=0$ in equation (14), we obtain

$$
g(\theta+\omega+\delta, 0, \delta)=g(\theta, 0, \delta) e^{\lambda(\omega+\delta)}
$$

Writing $g(\theta, \varepsilon, \delta)$ in Fourier series,

$$
g(\theta, \varepsilon, \delta)=\sum_{l \in \mathbb{Z}} g^{l}(\varepsilon, \delta) e^{2 \pi i l \theta},
$$

equation (18) yields, for each $l \in \mathbb{Z}$,

$$
g^{l}(0, \delta)\left(e^{2 \pi i l(\omega+\delta)}-e^{\lambda(\omega+\delta)}\right)=0 .
$$


Again, since $\lambda \neq 0$, we have $e^{2 \pi i l(\omega+\delta)}-e^{\lambda(\omega+\delta)} \neq 0$, so that $g^{l}(0, \delta)=0$ for all $l \in \mathbb{Z}$. Consequently, one has that $g(\theta, 0, \delta)=0$ for all $\delta$, which implies that for all $k \geq 1, g_{0 k}(\theta)=0$.

Finally, we consider the higher-order terms of equations (15) and (16). In the following, for a series $f(\varepsilon, \delta)=\sum_{m, n \geq 0} f_{m n} \varepsilon^{m} \delta^{n}$ we shall denote $[f(\varepsilon, \delta)]_{j, k}:=f_{j k}$. As we mentioned above, for $j, k$ such that $j+k \geq 1$ and $j \geq 1$, one can proceed inductively as follows. Assume that we have already computed $h_{j k}, g_{j k}$ with $j+k \leq N$ for some $N \geq 0$, and we need to compute $h_{j k}$ and $g_{j k}$ with $j+k=N+1$. We note that $h_{j k}$ is simply given by:

$$
h_{j k}(\theta)=\left[\theta+\omega+\delta+\varepsilon \operatorname{PRF}\left(\theta, \sum_{m, n \geq 0} g_{m n}(\theta) \varepsilon^{m} \delta^{n}\right)\right]_{j, k} .
$$

For $j \geq 1, k \geq 0$ we have

$$
h_{j k}(\theta)=\left[\operatorname{PRF}\left(\theta, \sum_{m, n \geq 0} g_{m n}(\theta) \varepsilon^{m} \delta^{n}\right)\right]_{j-1, k} .
$$

We note that the right-hand side of (19) depends only on $g_{m n}$ with $0 \leq m \leq j-1$ and $0 \leq n \leq k$, so that $m+n \leq j+k-1=N$, and thus they are already known.

Now, assume that we have already computed $g_{j k}$ with $j+k \leq N$ and $h_{j k}$ with $j+k \leq N+1$ and we want to compute $g_{j k}$ with $j+k=N+1$. From (16) one can easily see that $g_{j k}$ must satisfy

$$
g_{j k}(\theta+\omega)-g_{j k}(\theta) e^{\lambda \omega}=R_{j k}(\theta),
$$

where $R_{j k}$ is defined as

$$
\begin{aligned}
R_{j k}(\theta)= & -\left[\sum_{m, n \geq 0} g_{m n}\left(\sum_{r, s \geq 0} h_{r s}(\theta) \varepsilon^{r} \delta^{s}\right) \varepsilon^{m} \delta^{n}-g_{j k}(\theta+\omega) \varepsilon^{j} \delta^{k}\right]_{j, k} \\
& +\left[\left(\sum_{m, n \geq 0} g_{m n}(\theta) \varepsilon^{m} \delta^{n}\right) e^{\lambda(\omega+\delta)}-g_{j k}(\theta) \varepsilon^{j} \delta^{k} e^{\lambda \omega}\right]_{j, k} \\
& +\left[\varepsilon A R F\left(\theta, \sum_{m, n \geq 0} g_{m n}(\theta) \varepsilon^{m} \delta^{n}\right) e^{\lambda(\omega+\delta)}\right]_{j, k} .
\end{aligned}
$$


It is easy to see that, in fact,

$$
\begin{aligned}
& R_{j k}(\theta)=\left[-\sum_{\substack{0 \leq m \leq j \\
0 \leq n \leq k \\
m+n \leq j+k-1}} g_{m n}\left(\sum_{\substack{0 \leq r \leq j \\
0 \leq s \leq k \\
r+s \leq j+k}} h_{r s}(\theta) \varepsilon^{r} \delta^{s}\right) \varepsilon^{m} \delta^{n}\right]_{j, k} \\
& +\left[\sum_{\substack{0 \leq m \leq j \\
0 \leq n \leq k \\
m+n \leq j+k-1}} g_{m n}(\theta) \varepsilon^{m} \delta^{n} e^{\lambda(\omega+\delta)}\right]_{j, k} \\
& +\left[A R F\left(\theta, \sum_{\substack{0 \leq m \leq j-1 \\
0 \leq n \leq k \\
m+n \leq j+k-1}} g_{m n}(\theta) \varepsilon^{m} \delta^{n}\right) e^{\lambda(\omega+\delta)}\right]_{j-1, k}
\end{aligned}
$$

Note that $R_{j k}$ depends on $g_{m n}$ with $0 \leq m+n \leq j+k-1=N$ and $h_{m n}$ with $0 \leq m+n \leq j+k=N+1$ and, therefore, it is known. We point out that, in fact, in our setting, $R_{j k}$ does not depend on $h_{m n}$ with $m+n=N+1$ since $g_{00}(\theta)=0$. In order to solve equation (20) one can use Fourier series again. Indeed, if we write

$$
g_{j k}(\theta)=\sum_{l \in \mathbb{Z}} g_{j k}^{l} e^{2 \pi i l \theta}, \quad R_{j k}(\theta)=\sum_{l \in \mathbb{Z}} R_{j k}^{l} e^{2 \pi i l \theta}
$$

we can easily see that

$$
g_{j k}^{l}=\frac{R_{j k}^{l}}{e^{2 \pi i l \omega}-e^{\lambda \omega}}
$$

We remark that the denominator is always nonzero since $\lambda \neq 0$.

\section{B. Implementation of the method}

In this section we give some details of how we implemented the method described in Section III A. The main tool is computing series obtained by operating with two other series (adding, multiplying, etc.) and composing a given series with elementary functions (such as the exponential, sine, cosine,...). However, as one can see in equation (16), in our case one must also compute the series of the composition $g(h(\theta, \varepsilon, \delta), \varepsilon, \delta)$. We stress that $g(\theta, \varepsilon, \delta)$ is not known explicitly. In order to find the series of $g(h(\theta, \varepsilon, \delta), \varepsilon, \delta)$, we proceed 
as follows. Assume we have computed $g_{j k}(\theta), 0 \leq j+k \leq N$ for some $N$. Let us define

$$
g_{N}(\theta, \varepsilon, \delta)=\sum_{0 \leq j+k \leq N} g_{j k}(\theta) \varepsilon^{j} \delta^{k}
$$

Since $g_{N}$ is periodic with respect to $\theta$, we can also write it in Fourier series:

$$
\begin{aligned}
g_{N}(\theta, \varepsilon, \delta) & =\sum_{l \geq 0} \hat{g}_{N}^{l}(\varepsilon, \delta) \cos (2 \pi l \theta)+\bar{g}_{N}^{l}(\varepsilon, \delta) \sin (2 \pi l \theta) \\
& =\sum_{l \geq 0} \sum_{0 \leq j+k \leq N}\left(\hat{g}_{j, k}^{l} \cos (2 \pi l \theta)+\bar{g}_{j, k}^{l} \sin (2 \pi l \theta)\right) \varepsilon^{j} \delta^{k}
\end{aligned}
$$

The Fourier coefficients $\hat{g}_{j, k}^{l}$ and $\bar{g}_{j, k}^{l}$ are found numerically by computing the values of the function $g_{j, k}(\theta)$ for a discretization $\theta_{0}, \ldots, \theta_{n}$ and using the Fast Fourier Transform (FFT). In the examples below, we take $n=1024$ for the Fourier transform. In the numerical implementation, expansions (21) are truncated at a maximum Fourier index $l$. We choose this maximum Fourier index such that the tails of the Fourier expansion are small relatively to the order. More precisely, we fix two constants $E_{L}$ and $\chi$ and then for each $j, k \geq 0$ we choose $l_{\max }=l_{\max }(j, k)$ such that

$$
g_{j, k}(\theta)=\sum_{l=\left\lfloor 0.9 l_{\max }\right\rfloor}^{l_{\max }}\left(\left|\hat{g}_{j, k}^{l}\right|+\left|\bar{g}_{j, k}^{l}\right|\right)<\frac{E_{L}}{\chi^{j+k}} .
$$

We take $\chi<1$ so that as the order $j+k$ increases a larger error is tolerated, since for small values of $\varepsilon$ the contributions due to the terms $g_{j, k}(\theta)$ will be less significant. In the computations shown here we take $E_{L}=10^{-10}$ and $\chi=0.9$. In the following we denote

$$
L=\max _{0 \leq j+k \leq N} l_{\max }(j, k) .
$$

Following the convention that $\bar{g}_{j, k}^{l}=\hat{g}_{j, k}^{l}=0$ if $l>l_{\max }(j, k)$, equation (21) writes out as

$$
\begin{aligned}
g_{N}(\theta, \varepsilon, \delta) & =\sum_{l=0}^{L} \hat{g}_{N}^{l}(\varepsilon, \delta) \cos (2 \pi l \theta)+\bar{g}_{N}^{l}(\varepsilon, \delta) \sin (2 \pi l \theta) \\
& =\sum_{l=0}^{L} \sum_{0 \leq j+k \leq N}\left(\hat{g}_{j, k}^{l} \cos (2 \pi l \theta)+\bar{g}_{j, k}^{l} \sin (2 \pi l \theta)\right) \varepsilon^{j} \delta^{k} .
\end{aligned}
$$

Now, after computing the (truncated) Fourier series of $g_{N}$, we can write

$$
g_{N}(h(\theta, \varepsilon, \delta), \varepsilon, \delta)=\sum_{l=0}^{L} \hat{g}_{N}^{l}(\varepsilon, \delta) \cos (2 \pi l h(\theta, \varepsilon, \delta))+\bar{g}_{N}^{l}(\varepsilon, \delta) \sin (2 \pi l h(\theta, \varepsilon, \delta)),
$$


and compute the cosine and sine series with methods of automatic differentiation:

$$
\begin{aligned}
& \cos (2 \pi l h(\theta, \varepsilon, \delta))=: c^{l}(\theta, \varepsilon, \delta)=\sum_{j, k \geq 0} c_{j, k}^{l}(\theta) \varepsilon^{j} \delta^{k} \\
& \sin (2 \pi l h(\theta, \varepsilon, \delta))=: \quad s^{l}(\theta, \varepsilon, \delta)=\sum_{j, k \geq 0} s_{j, k}^{l}(\theta) \varepsilon^{j} \delta^{k} .
\end{aligned}
$$

Finally, we just need to compute the series of the following products for each $l$, which can be done again using methods of automatic differentiation:

$$
\begin{aligned}
& \hat{g}_{N}^{l}(\varepsilon, \delta) c^{l}(\theta, \varepsilon, \delta)=a^{l}(\theta, \varepsilon, \delta)=: \sum_{j, k \geq 0} a_{j, k}^{l}(\theta) \varepsilon^{j} \delta^{k} \\
& \bar{g}_{N}^{l}(\varepsilon, \delta) s^{l}(\theta, \varepsilon, \delta)=b^{l}(\theta, \varepsilon, \delta)=: \sum_{j, k \geq 0} b_{j, k}^{l}(\theta) \varepsilon^{j} \delta^{k} .
\end{aligned}
$$

The series of $g_{N}(h(\theta, \varepsilon, \delta), \varepsilon, \delta)$ is then given by

$$
g_{N}(h(\theta, \varepsilon, \delta), \varepsilon, \delta)=\sum_{l=0}^{L} a^{l}(\theta, \varepsilon, \delta)+b^{l}(\theta, \varepsilon, \delta)=\sum_{j, k \geq 0}\left(\sum_{l=0}^{L} a_{j, k}^{l}(\theta)+b_{j, k}^{l}(\theta)\right) \varepsilon^{j} \delta^{k}
$$

we neglect the terms with $j+k>N$ since they will be modified when computing the series of $g_{N+1}(h(\theta, \varepsilon, \delta), \varepsilon, \delta)$, and so on.

Remark III.1 In the practical implementation, we choose L to be at most 25. The reason is that the error in the the $c^{l}$ and $s^{l}$ series (23) increases with $l$. To decrease this error, one needs to compute more orders of these expansions, that is, to increase $N$. This, in its turn, increases the maximum $L$ needed to control the error of the Fourier expansions (21), ending in vicious circle.

Finally, we point out that we need to compute as well the series of $\operatorname{PRF}(\theta, g(\theta, \varepsilon, \delta))$ and $A R F(\theta, g(\theta, \varepsilon, \delta))$, see the invariance equations (15) and (16). In the example below, we will know these functions explicitly, so that they can be computed using automatic differentiation tools again. However, in realistic models, the PRF and ARF are computed numerically as seen in [5]. In this case, one has these functions expressed as Fourier-Taylor series:

$$
\operatorname{PRF}(\theta, \sigma)=\sum_{n=0}^{n_{\max }} P R F_{n}(\theta) \sigma^{n}, \quad A R F(\theta, \sigma)=\sum_{n=0}^{n_{\max }} A R F_{n}(\theta) \sigma^{n}
$$

One just needs to compute the series of $(g(\theta, \varepsilon, \delta))^{n}$ for $n=0, \ldots, n_{\max }$, which can be done with standard methods, and then one can find the series of $\operatorname{PRF}(\theta, g(\theta, \varepsilon, \delta))$ and $\operatorname{ARF}(\theta, g(\theta, \varepsilon, \delta))$. 


\section{Computation of Arnold tongues}

Once we have computed the series $g(\theta, \varepsilon, \delta)$ (the parameterization of the invariant curve) and $h(\theta, \varepsilon, \delta)$ (the dynamics inside it), we can proceed to look for the Arnold tongue of rotation number $p / q$. To that aim, we consider the function:

$$
\mathcal{F}_{p / q}(\theta, \varepsilon, \delta)=\left(h^{q}(\theta, \varepsilon, \delta)-\theta-p, \partial_{\theta}\left(h^{q}(\theta, \varepsilon, \delta)\right)-1\right)
$$

Given a fixed $\varepsilon$, we look for $(\theta, \delta)=(\theta(\varepsilon), \delta(\varepsilon))$ such that $\mathcal{F}_{p / q}(\theta(\varepsilon), \varepsilon, \delta(\varepsilon))=0$, which ensures that $\left(\theta(\varepsilon), g(\theta(\varepsilon))\right.$ is a saddle-node bifurcation point of the function $F_{\varepsilon, \frac{p}{q}+\delta(\varepsilon)}$ defined in (10). This equation can be solved using Newton's method. We point out that, having computed the coefficients of the series $h(\theta, \varepsilon, \delta)$, the computation of the derivative of $h$ with respect to $\delta$ is trivial. The derivative of $h$ with respect to $\theta$ is computed by means of the FFT algorithm, using again a discretization of the function at $n=1024$ points.

After computing $(\theta(\varepsilon), \delta(\varepsilon))$, we change $\varepsilon$ by some small amount $\Delta \varepsilon$ and follow a continuation method to obtain a good initial approximation of $(\theta(\varepsilon+\Delta \varepsilon), \delta(\varepsilon+\Delta \varepsilon))$. This is done using one step of Newton's method. Then, we start again the procedure described above to find the solution with the desired accuracy.

We increase $\varepsilon$ up to some maximum value $\varepsilon_{\max }$ so that the invariance equations (13) and (14) are satisfied up to some error $E_{\text {inv }}$. That is, we choose $\varepsilon_{\max }$ to be the maximum value of $\varepsilon$ such that for all $\varepsilon \leq \varepsilon_{\max }$ :

$$
\sup _{\theta \in[0,1)}|h(\theta, \varepsilon, \delta(\varepsilon))-\theta+\omega+\delta(\varepsilon)+\varepsilon P R F(\theta, g(\theta, \varepsilon, \delta(\varepsilon)))|<E_{\text {inv }} .
$$

and:

$$
\sup _{\theta \in[0,1)}\left|g(h(\theta, \varepsilon, \delta(\varepsilon)), \varepsilon, \delta(\varepsilon))-[g(\theta, \varepsilon, \delta(\varepsilon))+\varepsilon A R F(\theta, g(\theta, \varepsilon, \delta(\varepsilon)))] e^{\lambda(\omega+\delta(\varepsilon))}\right|<E_{\text {inv }} .
$$

In the computations presented here we take $E_{\text {inv }}=10^{-10}$.

For $\varepsilon>\varepsilon_{\max }$ we can continue the Arnold tongues just by looking for a saddle-node bifurcation point of the 2D-map $F_{\varepsilon, p / q+\delta}^{q}(\theta, \sigma)$ defined in (10). That is, given a certain $\varepsilon>\varepsilon_{\max }$, we look for a point $(\theta(\varepsilon), \sigma(\varepsilon), \delta(\varepsilon))$ such that:

$$
\begin{aligned}
F_{\varepsilon, p / q+\delta(\varepsilon)}^{q}(\theta(\varepsilon), \sigma(\varepsilon)) & =0, \\
\operatorname{det}\left(D F_{\varepsilon, p / q+\delta(\varepsilon)}^{q}(\theta(\varepsilon), \sigma(\varepsilon))-\mathrm{Id}\right) & =0 .
\end{aligned}
$$

We perform a Newton method to obtain such a point $(\theta(\varepsilon), \sigma(\varepsilon), \delta(\varepsilon))$, taking the seed $\left(\theta(\varepsilon \max ), \sigma\left(\varepsilon_{\max }\right), \delta\left(\varepsilon_{\max }\right)\right)$. 
We point out that for $\varepsilon>\varepsilon_{\max }$ we cannot ensure that the points $(\theta(\varepsilon), \sigma(\varepsilon))$ lie on an invariant curve anymore.

Remark III.2 This method has a particular drawback for our interests. If one wants to deal with realistic synaptic inputs, one should consider $p / q<1 / 20$. To find the series of $h^{q}(\theta, \varepsilon, \delta)$ with $q$ large, the computation time can be too long, and even the accuracy of the series too bad (that is, $\varepsilon_{\text {max }}$ too small). One possible solution is computing a normal form of $h(\theta, \varepsilon, \delta)$ in terms of $\delta$, as is done in [20].

\section{Numerical aspects}

For the numerical integration we have used a Runge-Kutta method of order $7 / 8$ with a fixed tolerance of $10^{-12}$. To track the invariant curves for different parameter values we have used continuation methods as explained in [24]. In the practical implementation of the procedure explained in Section III, we have used automatic differentiation tools, see for instance [23] and [25]. To compute the FFT we have used the fftw 3 library (see http://www.fftw.org/).

\section{Application to the canonical type II oscillator}

In this section we show the results obtained with the implementation of the methods presented in Section III. We apply them to the canonical example (1) studied in Section II. As a showcase, we take $\alpha=5$ and $a=1$, so that the underlying limit cycle of the continuous system is strongly hyperbolic and the isochrons are slightly tilted. Despite being a conservative parameter choice, we already detect differences between the 1D and the 2D entrainment maps. Unfortunately, for $\alpha<<1$ (the more realistic situation, explored in [5]), in which we expect more dramatic differences between the two entrainment maps, the convergence of the methods worsens.

We first show some invariant curves of the 2D map (10) with $\omega=1 / 50$ for different values of $\varepsilon$ obtained with the Newton-like method (see Appendix V) and the Taylor expansion method. On the one hand, we plot the invariant curves in variables $(\theta, \sigma)$ (see Figure 4). On the other hand, we plot the same curves in variables $(x, y)=K(\theta, \sigma)$, where $K$ is the function defined in (3) (see Figure 5). As we mentioned above, in both methods the maximal value of $\varepsilon$ that we can reach keeping a low error in the invariance equations is not completely satisfactory, since we are not able to see invariant curves close to the breakdown. However, 
one can see the evolution of these invariant curves as $\varepsilon$ increases. This evolution is much more visible in $(\theta, \sigma)$ variables, see Figure 4.

Next, we fix $p / q=1 / 3$, and we plot the corresponding Arnold tongue (see Figure 6) for $\varepsilon<\varepsilon_{\max }$. We also take some points on the parameter line $\varepsilon=0.2$ and plot the corresponding invariant curves in Figure 7 . We can observe a saddle-node bifurcation of periodic orbits: we start having two 1/3-periodic orbits (one attracting and the other repelling) that approach each other until they collide, giving rise to a single $1 / 3$ periodic orbit of saddle-node type. Beyond this parameter value, rational dynamics is no longer observed (see Figure 7d).

Finally, we show some Arnold tongues of map $F_{\varepsilon, \omega}$. We first plot some tongues for low values of $p / q$ (see Figure 8). We also indicate the value of $\varepsilon_{\max }$, that is the value of $\varepsilon$ such that $E_{\mathrm{inv}}<10^{-10}$. In Figure 9 we compare some Arnold tongues (for low values of $p / q$ ) corresponding to the 2D entrainment map $F_{\varepsilon, \omega}$ (in red) and the 1-dimensional map (9) (in blue). One can see that, the higher $q$ is, the more the tongues of the $1 \mathrm{D}$ and $2 \mathrm{D}$ maps differ. We expect that for realistic values of $p / q$ (for instance, $p / q=1 / 50$ ) these differences will be significant. However, working with double precision does not allow us to distinguish between the two boundaries of the Arnold tongues for high values of $q$ (see Figure 10). This is due to the fact that the order of contact of the tongues is of $\varepsilon^{q}$, see [20]. In order to be able to compute the corresponding tongues in these cases, one should work with higher-precision arithmetics or with normal forms, as is done in [20].

\section{A. Simulation results}

Up to now, we have shown results coming from rigorous computations that rely on the analytico-numerical method developed in Section III. Unfortunately, the methods and the required tolerance ar very demanding and we cannot visualize all the dynamics as the parameters $\varepsilon$ or $\omega$ change up to the invariant curve breaks up. In this section, we present simulations that show how the invariant dynamic varies up to higher values of the parameters. In Figures 11 and 12 we present simulations in which we can see the evolution of the asymptotic attractors when one changes the stimulus amplitude $\varepsilon$ or the relative period of the stimulus $T_{s} / T_{0}$, respectively. Another way to visualize the predictive power of both entrainment maps is to compare the phase dynamics $\left(\left\{\theta_{n}\right\}_{n}\right)$ of the exact map with those of the 1D and the 2D maps. In Figure 13, we present the iterates of the phase for the same values than in Figure 11. Altogether, we can draw the following observations: 


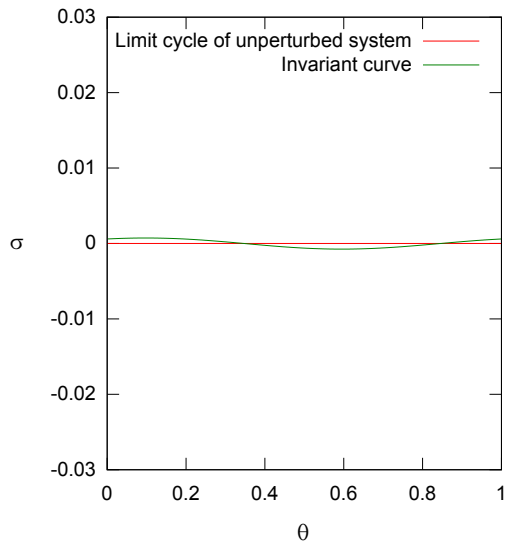

(a) Newton-like method, $\varepsilon=0.001$.

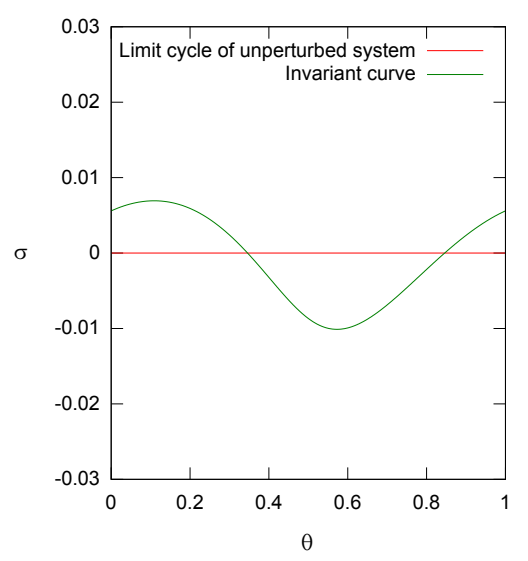

(c) Newton-like method, $\varepsilon=0.011$.

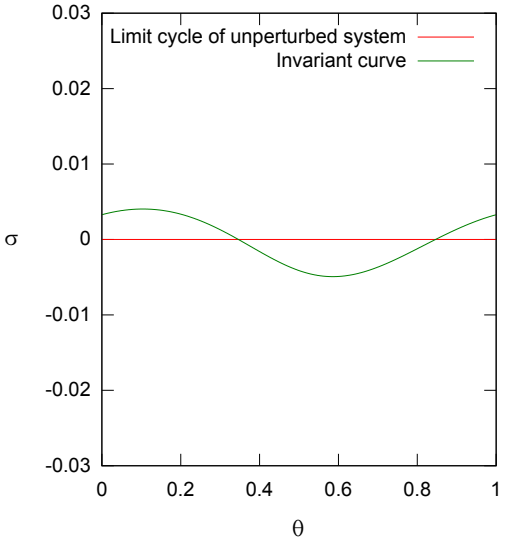

(b) Newton-like method, $\varepsilon=0.006$.

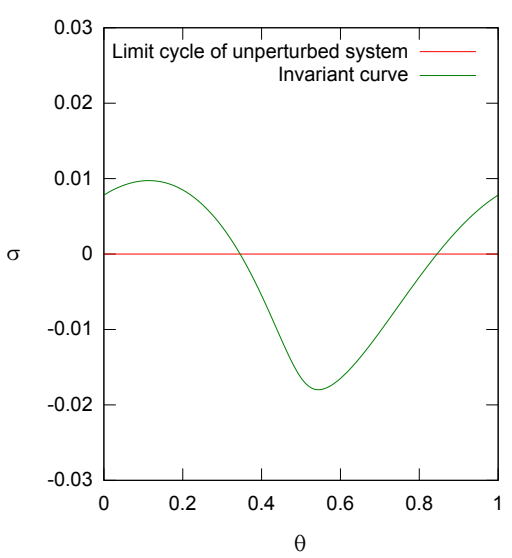

(d) Newton-like method, $\varepsilon=0.0165$.

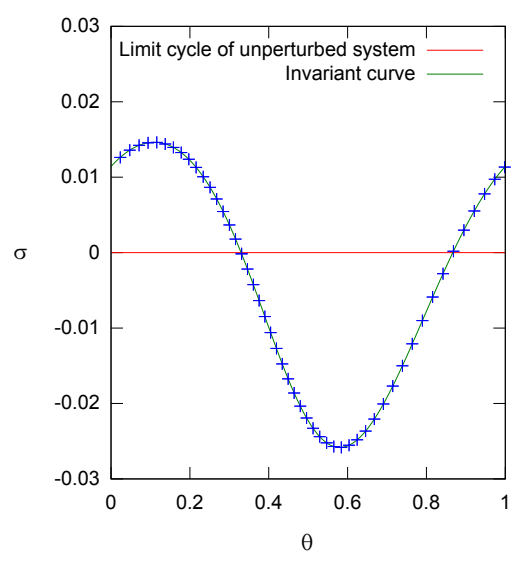

(e) Taylor expansion method. $\varepsilon=0.021$ and $\delta=0.0004$ are chosen so that the invariant curve lies on the boundary of the $1 / 50$-Arnold tongue. In blue, the 1/50-periodic orbit.

Figure 4: Invariant curves of the map $F_{\varepsilon, \omega}$ and the unperturbed limit cycle. We consider system (1) with $\alpha=5, a=1$ and perturb it with a periodic pulse stimulus with frequency $\omega=1 / 50$ and amplitude $\varepsilon$. The results are shown on the plane $(\theta, \sigma)$. 


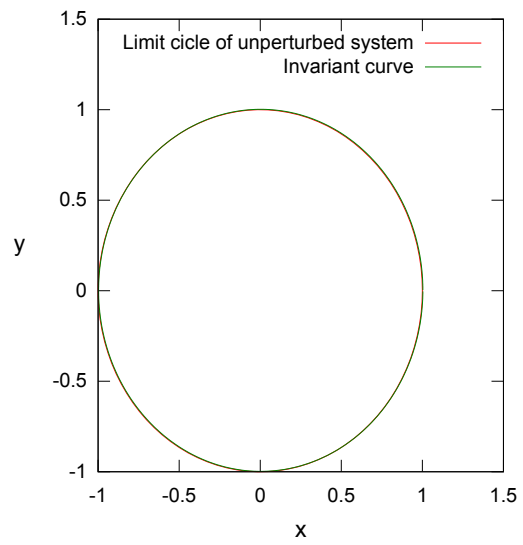

(a) Newton-like method, $\varepsilon=0.001$.

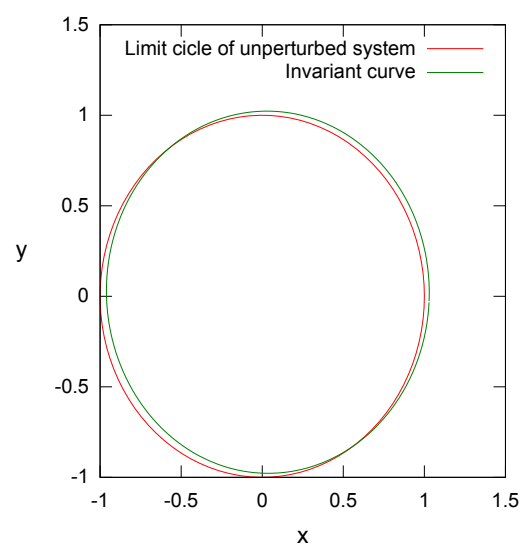

(c) Newton-like method, $\varepsilon=0.011$.

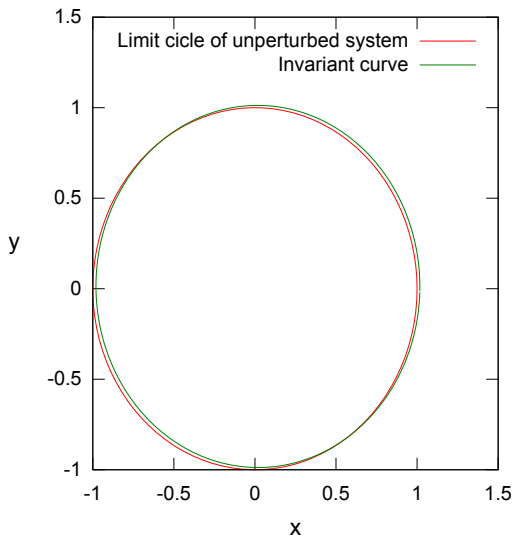

(b) Newton-like method, $\varepsilon=0.006$.

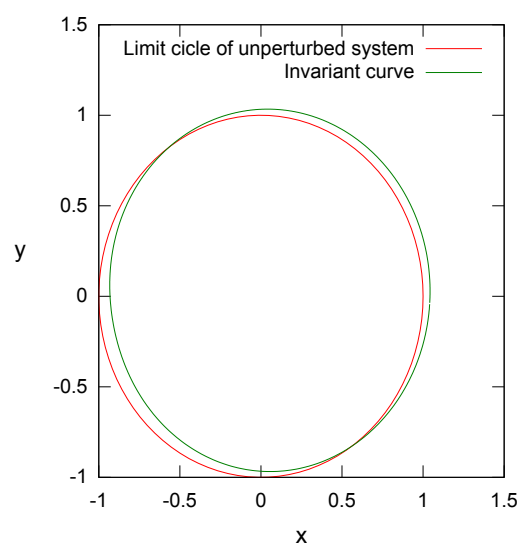

(d) Newton-like method, $\varepsilon=0.0165$.

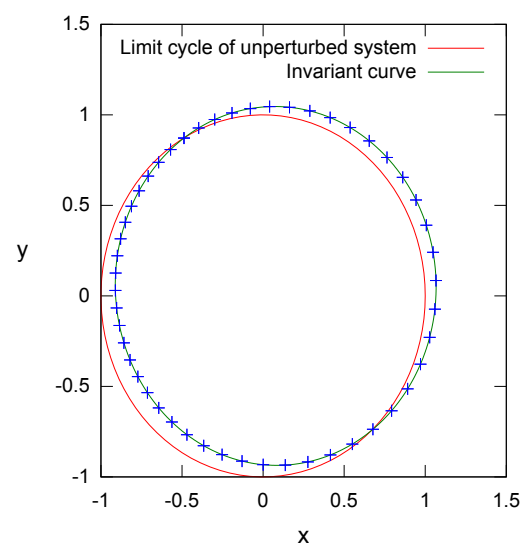

(e) Taylor expansion method. $\varepsilon=0.021$ and $\delta=0.0004$ are chosen so that the invariant curve lies on the boundary of the $1 / 50$-Arnold tongue. In blue, the 1/50-periodic orbit.

Figure 5: Invariant curves of the map $F_{\varepsilon, \omega}$ and the unperturbed limit cycle. We consider system (1) with $\alpha=5, a=1$ and perturb it with a periodic pulse stimulus with frequency $\omega=1 / 50$ and amplitude $\varepsilon$. The results are shown on the plane $(x, y)=K(\theta, \sigma)$. 


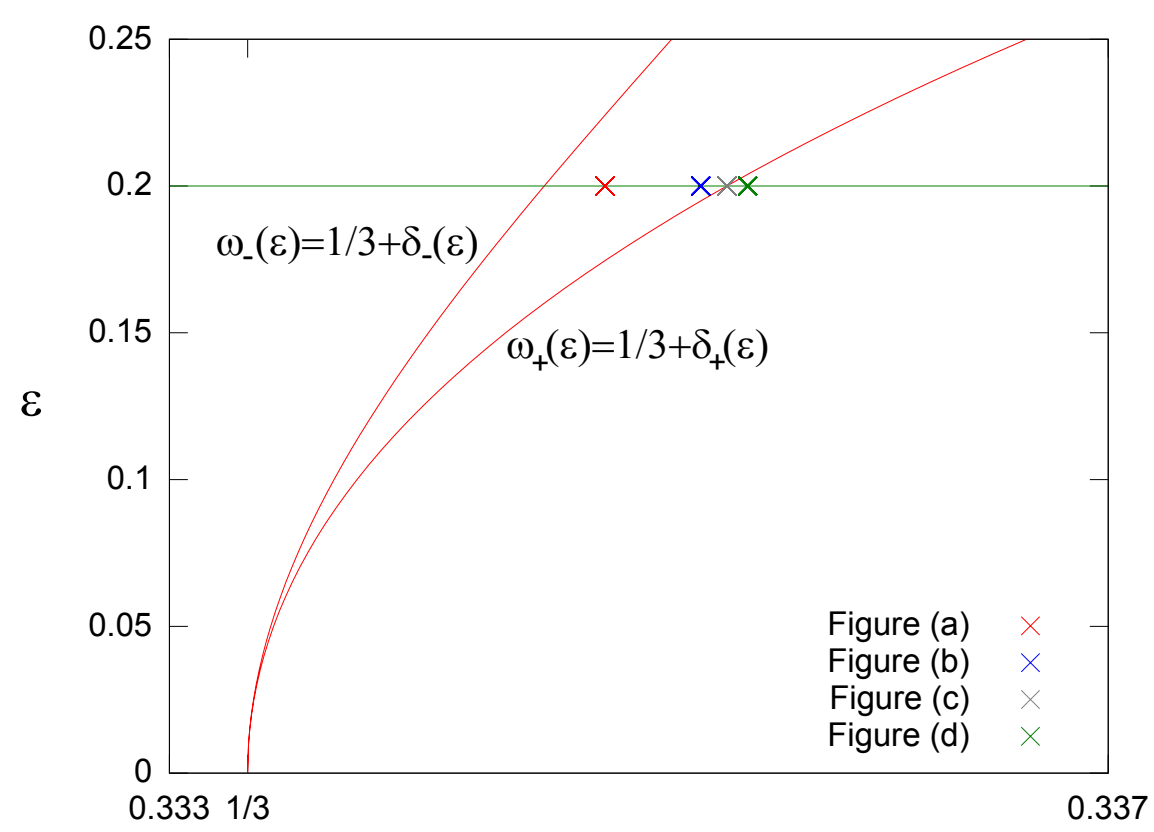

$\omega$

Figure 6: The 1/3-Arnold tongue for the map $F_{\varepsilon, \omega}$. We consider system (1) with $\alpha=5, a=1$. The map $F_{\varepsilon, \omega}$ has period-3 orbits inside the tongue, which is delimited by the curves $\omega_{ \pm}(\varepsilon)=1 / 3+\delta_{ \pm}(\varepsilon)$. Each cross on the line $\varepsilon=0.2$ corresponds to a panel in Figure 7 .

- The three maps seem to have an invariant curve under weak perturbations (small amplitude or large stimulus period) that breaks down to give rise to a fixed-point attractor when either $\varepsilon$ or $\omega$ are big enough. Under weak perturbations (for instance, $\epsilon=0.001$ in Figures 11 and 13), both the 1D and the 2D map give a good prediction.

- Further increasing $\epsilon$ or $\omega$, we find a range (for instance, $\epsilon=0.017,0.033$ in Figures 11 and 13) where the exact invariant curve persists and displaces from the original position of the limit cycle; here, the 2D map is able to track this displacement whereas the 1D map not. However, both maps give good approximations of the phase. We conclude that, in this range, the 1D map looses predictive power about the amplitude but not about the phase.

- This breakdown takes place at different perturbation levels according to the map considered: first, the exact map and later on, the 2D and the 1D maps. In this case, both the 2D map and the 1D map fail to capture the phase, but the 2D map, at first iterates, keeps better track of the bumpy behaviour of the exact phase.

- Finally, for even stronger perturbations (for instance, $\epsilon=0.079,0.089$ in Figures 11 and 13), the 


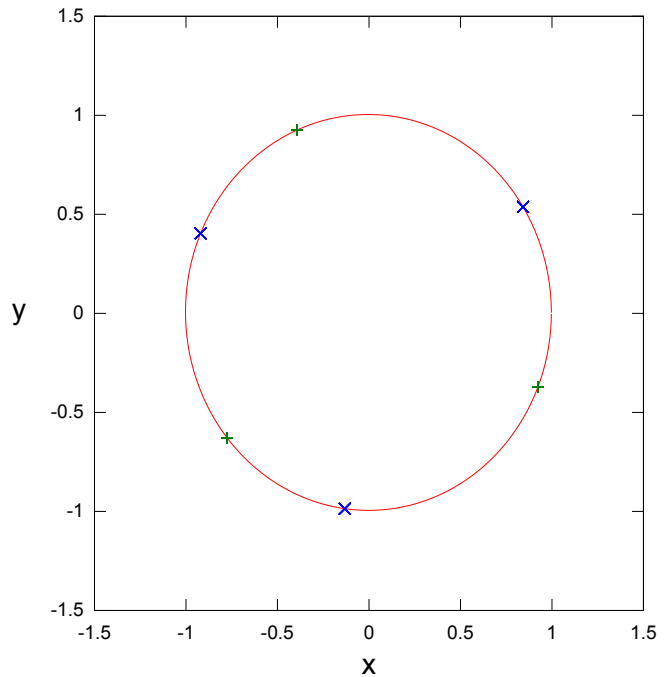

(a) $\omega=1 / 3+0.0015233263$. One has two

$1 / 3$-periodic orbits.

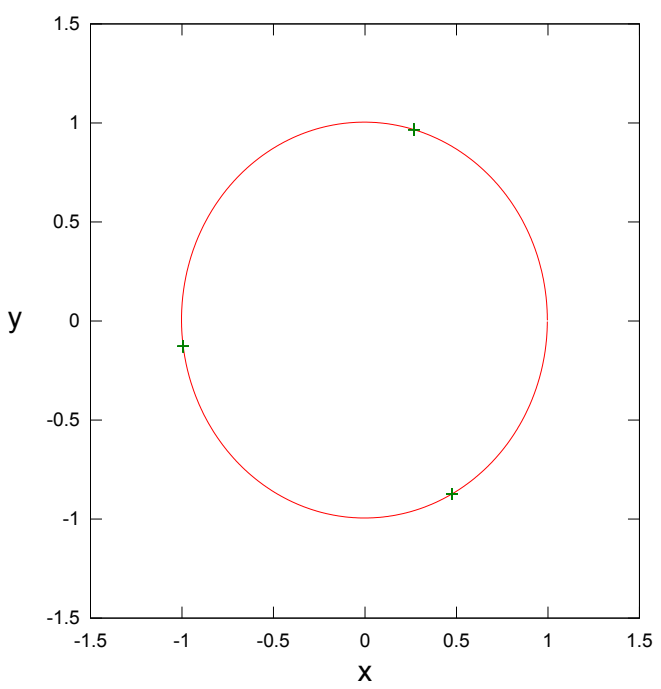

(c) $\omega=1 / 3+0.0020418188$. The two periodic orbits collide, giving rise to a single saddle-node 1/3-periodic orbit.

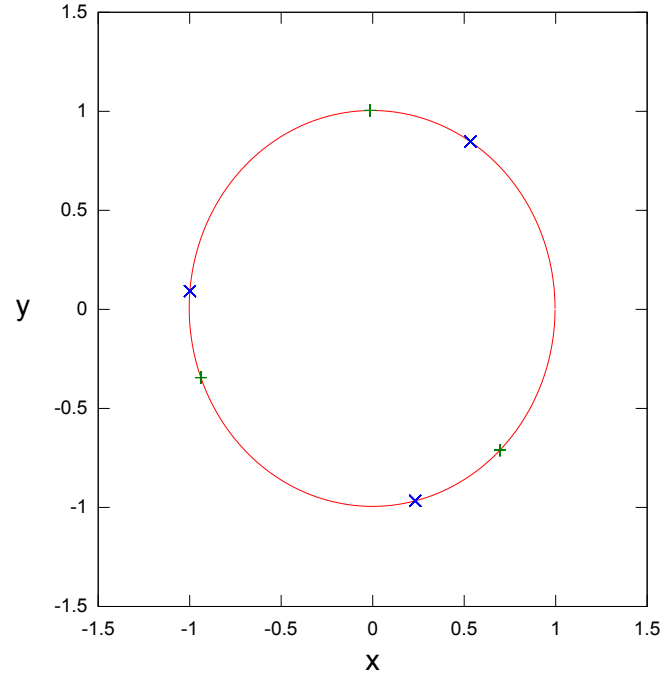

(b) $\omega=1 / 3+0 .+0.00193$. One has two $1 / 3$-periodic orbits.

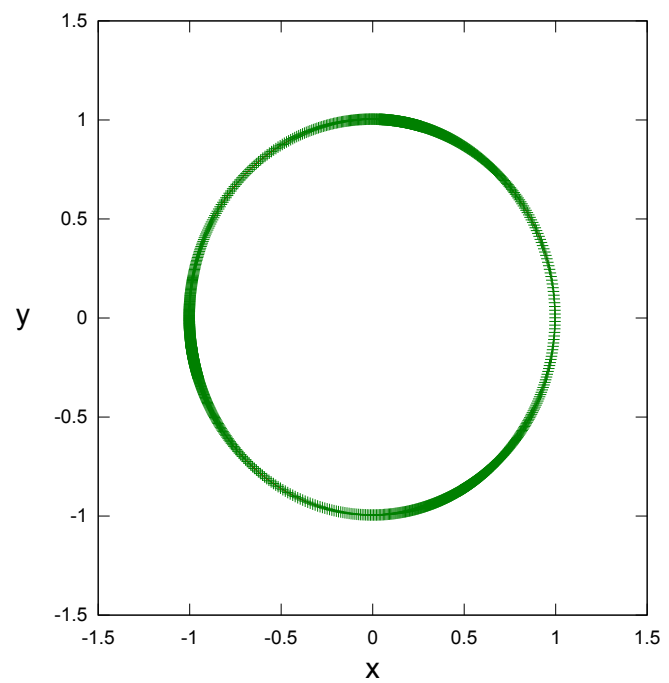

(d) $\omega=1 / 3+0.00213 .1500$ iterations of a point inside the invariant curve. Apparent irrational dynamics.

Figure 7: Dynamics of $F_{\varepsilon, \omega}$ across the 1/3-Arnold Tongue. Invariant curves of the map $F_{\varepsilon, \omega}$ with $\alpha=5$, $a=1$ and $\varepsilon=0.2$ in the plane $(x, y)$. We consider four different values of $\omega$, corresponding to the four situations marked on Figure 6. The values of $\omega$ for panels (a) and (b) belong to the interior of the 1/3-Arnold tongue and show two periodic orbits of period 3. Panel (c) corresponds to the boundary of the Arnold tongue where a saddle-node bifurcation occurs. Panel (d) corresponds to a value of $\omega$ out of the Arnold tongue; we have lost the 3-periodic orbit and observe apparent irrational dynamics. 


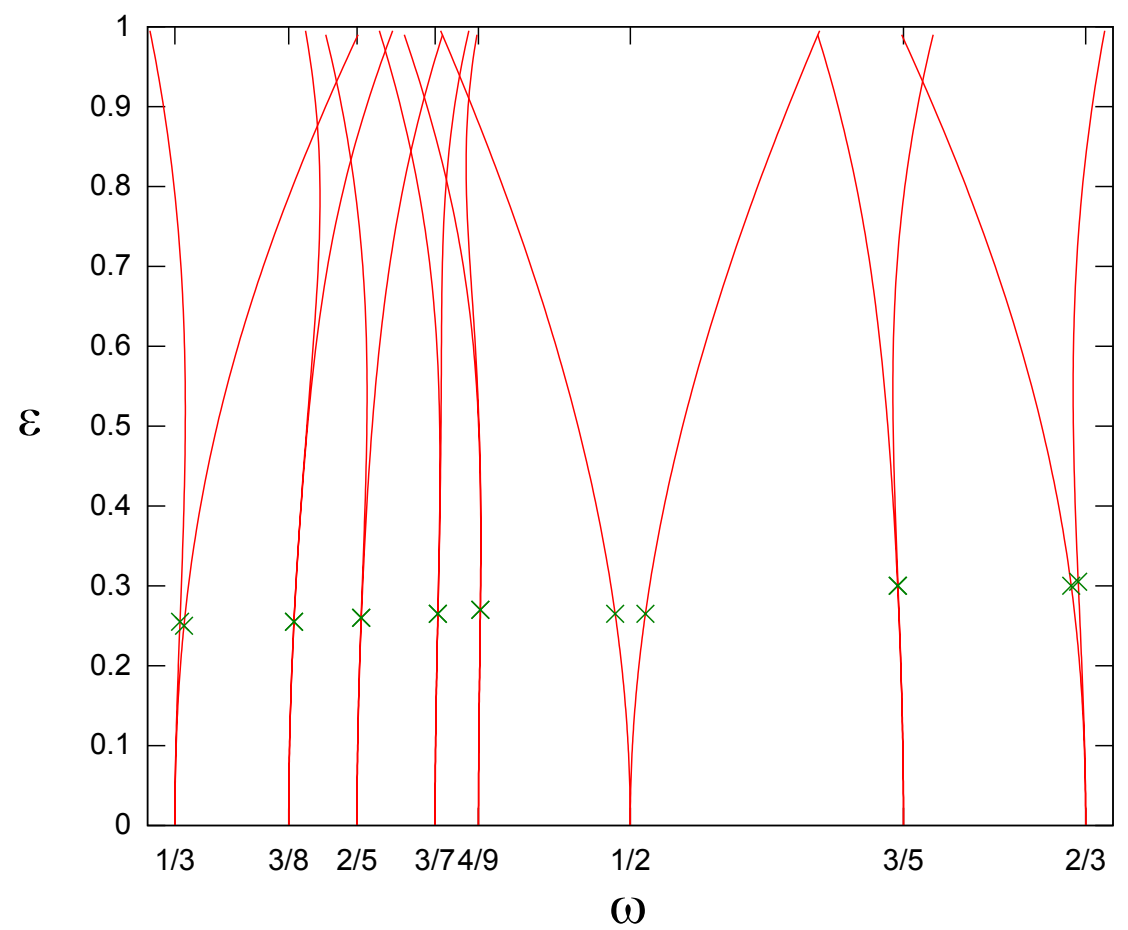

Figure 8: Arnold tongues for the map $F_{\varepsilon, \omega}$ with with $\alpha=5, a=1$. Green crosses indicate the value of $\varepsilon_{\max }$ of each tongue (see Section III C).

invariant curve of the 2D map also breaks down. Both the exact map and the 2D map spiral around a focus beyond this break-down bifurcation value and the 2D map is able to predict oscillations of the phase. In this regime, the 1D map (which can never break down) is not predicting anymore the exact phase.

- The fixed point of the 2D map gives a better approximation of the fixed point of the exact map. Thus, for big enough perturbations, both the 1D map and the 2D map predict a phase-locking, but the predicted phase of the 1D map is less accurate.

\section{Discussion}

We have developed an analytico-numerical scheme to gain more insight into the dynamics of the phaseamplitude entrainment map defined in (8). Adapting methods from the literature on 2D maps, we provide two alternatives to compute invariant curves of the entrainment map as well as their intrinsic dynamics. We then apply them to a specific minimal model. 
We have validated numerically the existence of invariant curves up to some perturbation level $\varepsilon_{\max }$ for different stimulation frequencies. Since we have used a tolerance $10^{-10}$ to compute the boundaries of the Arnold tongues, this $\varepsilon_{\max }$ is a rigorous lower bound for the existence of the invariant curve.

The methodology introduced provides a better understanding of the dynamics on the invariant curves, while distinguishing parameter regions with rational dynamics from those with irrational by means of the Arnold tongues. In particular, we have described the saddle-node bifurcation of periodic orbits that the system undergoes when crossing the boundaries of these Arnold tongues. Compared to parallel results obtained with the 1D entrainment map (9), we see that the Arnold tongues are slightly different. However, for numerical reasons, we have kept at parameter values that were not favorable to show a clear distinction. From the results in [5], we know that the conditions favorable to the loss of validity of the asymptotic-phase hypothesis are weak hyperbolicity and high periodic stimulation. The results shown here are obtained for strong hyperbolic limit cycles $(a=5)$ and slow frequency pulsatile stimuli, which can be seen by observing the $\omega$ values of the computed Arnold tongues (see Figures 8 and 6).

By comparing the entrainment maps (9) and (8) with the exact entrainment map, we have validated our hypothesis that the 2D map would provide better predictions for strong or high-frequency stimuli. In particular, we have found ranges where the $2 \mathrm{D}$ map is able to track the displacement of the invariant whereas the 1D map not, an intermediate range where the invariant curve of the exact map breaks down and both the 2D map and the 1D map fail to capture the phase, and another range, beyond the breakdown of the invariant curves, for stronger perturbations, were the invariant curve of the 2D map provides again a good prediction of the exact phase whereas the 1D map fails to predict the exact phase.

This paper being a proof-of-concept of the methodology to study entrainment maps for phase-amplitude equations, it opens a wide range a future directions. A first challenge would be to improve the implementation of these methods in order to be able to achieve more realistic values of $p / q$. As we pointed out above, this should be attainable by performing a normal form procedure so that the map $F_{\varepsilon, \omega+\delta}$ is in the simplest form, namely, to be able to write $F_{\varepsilon, \omega+\delta}$ in powers of $\varepsilon$ with all coefficients of order $n, 0<n<q$, depending only on $\delta$. Then, the equations to find the boundaries of the Arnold tongues would be also simplified (see [20, Prop. 2.9]), being able to easily distinguish between the two boundaries even for higher values of $q$, which are more realistic in the neuroscience paradigm. In addition, one could use higher-precision arithmetics.

Another goal would consist of computing the invariant curves for values of $\varepsilon$ that are close to the breakdown. In this direction, similarly as in [20], it would be interesting to compute the curves in the $(\omega, \varepsilon)$ space until 
which an invariant curve exists, and thus confirm the breakdown phenomenon observed in the simulations of Figures 11 and 12.

Focusing on the interpretation of these results in the context of neuroscience (see also [1, Section 10.1.9], for a similar discussion with the 1D PRC map), the Arnold tongues inform about the strength and periodicity of periodic pulse stimuli in order to achieve or not an entrainment of the cell to the stimulus. The differences between the 1D map and the 2D map predictions shown in Figure 10 are not striking for small $\varepsilon$ and "large" $\omega$, but they show the trend of an increasing dissimilarity as $\varepsilon$ increases and $\omega=T_{s} / T_{0}$ decreases (that is, when the stimulation period $T_{s}$ decreases). In particular, for realistic $\varepsilon$ and $T_{s}$, one expects stronger differences between the two predictions, meaning that an external control exerted on a neuron model might not have the synchronization properties forecasted by the 1D map. Our results show differences between the intervals predicted by the 1D map and the 2D map (supposedly closer to the actual one), corresponding to the interior of the respective $\omega$ Arnold tongues, and reinforce the warnings about the validity of this control using only 1D maps.

Natural continuations of our exploration will be the implementation of these methods to type I oscillators or to more realistic models. One could also try to study other type of pulsatile stimuli (for instance nonperiodic or noisy), other protocols of stimulation (two different periods of stimulation, pulse train, etc.) or heterogeneous input strengths. Doing so, one would obtain another map different from (8), but the same questions could be posed. Compared to the system studied in this paper, we would have to rely on numerical simulations since we would not have available the analytical expression of PRF and ARF. Although the paradigm that we analyze in this paper is apparently restrictive, see Remark II.1, we think that the conclusions obtained are robust for less restrictive inputs; for instance, a stochastic input with a stationary mean firing rate and strength distribution would be a candidate to numerically replicate similar results.

It is worth to note that this discussion is not only valid in the context of neuroscience. In fact, this was only our leitmotiv and we have brought the problem to a more mathematical (and so, generic) framework. Not surprisingly, this methodology can be applied to any model in which we have an oscillator, namely a limit cycle. As far as we know, only PRCs have been systematically used in other fields like electrical circuits, see [26], or cellular oscillators, see [27] and [28], which gives promising avenues for future work. 


\section{Acknowledgements}

This work has been partially funded by the Spanish grant MINECO-FEDER-UE MTM-2015-71509-C2-2$\mathrm{R}$ and the the Catalan Grant 2017SGR1049. We also acknowledge the use of the UPC Dynamical Systems group's cluster for research computing (https://dynamicalsystems.upc.edu/en/computing/). We are grateful to Marta Canadell and Àlex Haro for fruitful discussions on the Newton-like method.

[1] E. M. Izhikevich. Dynamical systems in neuroscience: the geometry of excitability and bursting. Computational Neuroscience. MIT Press, Cambridge, MA, 2007.

[2] B. Ermentrout and D.H. Terman. Mathematical Foundations of Neuroscience. Springer, 2010.

[3] Nathan W. Schultheiss, Astrid A. Prinz, and Robert J. Butera. Phase Response Curves in Neuroscience Theory, Experiment, and Analysis. Springer Series in Computational Neuroscience book series (NEUROSCI, volume 6). Springer, Cambridge, MA, 2012.

[4] A. Guillamon and G. Huguet. A computational and geometric approach to phase resetting curves and surfaces. SIAM J. Appl. Dyn. Syst., 8(3):1005-1042, 2009.

[5] O. Castejón, A. Guillamon, and G. Huguet. Phase-amplitude response functions for transient-state stimuli. The Journal of Mathematical Neuroscience (JMN), 3(1):1-26, 2013.

[6] K. C. A. Wedgwood, K. K. Lin, R. Thul, and S. Coombes. Phase-amplitude descriptions of neural oscillator models. J. Math. Neurosci, 3(2), 2013.

[7] S. Shirasaka, W. Kurebayashi, and Nakao H. Phase-amplitude reduction of transient dynamics far from attractors for limit-cycling systems. Chaos, 27:119-, 2017.

[8] Bharat Monga and Jeff Moehlis. Optimal phase control of biological oscillators using augmented phase reduction. Biological Cybernetics, 113(1):161-178, Apr 2019.

[9] Dan Wilson and Bard G. Ermentrout. Augmented phase reduction of (not so) weakly perturbed coupled oscillators. SIAM Review, 61(2):277-315, 2019.

[10] A. Rabinovitch and M. Friedman. Fixed points of two-dimensional maps obtained under rapid stimulations. Phys. Lett. A, 355(4-5):319-325, 2006.

[11] H.M. Osinga and J. Moehlis. Continuation-based computation of global isochrons. SIAM J. Appl. Dyn. Syst., 9(4):1201-1228, 2010.

[12] Gemma Huguet and Rafael de la Llave. Computation of limit cycles and their isochrons: Fast algorithms and their convergence. SIAM J. Appl. Dyn. Syst., 12(4):1763-1802, 2013.

[13] Peter Langfield, Bernd Krauskopf, and Hinke M. M. Osinga. Forward-time and backward-time isochrons and their interactions. SIAM J. Appl. Dyn. Syst., 14(3):1418-1453, 2015. 
[14] Gemma Huguet, Alberto Pérez-Cervera, and T.M. Seara. A geometric approach to phase response curves and its numerical computation through the parameterization method. arXiv:1809.07318, 2010.

[15] Jérémie Lefebvre, Axel Hutt, Jean-François Knebel, Kevin Whittingstall, and Micah M. Murray. Stimulus statistics shape oscillations in nonlinear recurrent neural networks. Journal of Neuroscience, 35(7):2895-2903, 2015.

[16] Alagapan S, Schmidt SL, Lefebvre J, Hadar E, Shin HW, and FrÓ§hlich F. Modulation of cortical oscillations by low-frequency direct cortical stimulation is state-dependent. PLoS Biol, 14(3):e1002424, 2016.

[17] Christoph S. Herrmann, Micah M. Murray, Silvio Ionta, Axel Hutt, and Jérémie Lefebvre. Shaping intrinsic neural oscillations with periodic stimulation. Journal of Neuroscience, 36(19):5328-5337, 2016.

[18] Casey O. Diekman and Amitabha Bose. Entrainment Maps: A New Tool for Understanding Properties of Circadian Oscillator Models. Journal of Biological Rhythms, 2016.

[19] Casey O. Diekman and Amitabha Bose. Reentrainment of the circadian pacemaker during jet lag: East-west asymmetry and the effects of north-south travel. Journal of Theoretical Biology, 437:261-285, 2018.

[20] H. W. Broer, C. Simó, and J. C. Tatjer. Towards global models near homoclinic tangencies of dissipative diffeomorphisms. Nonlinearity, 11(3):667, 1998.

[21] Marta Canadell and Àlex Haro. A Newton-like Method for Computing Normally Hyperbolic Invariant Tori, pages 187-238. Springer International Publishing, Cham, 2016.

[22] X. Cabré, E. Fontich, and R. de la Llave. The parameterization method for invariant manifolds. III. Overview and applications. J. Differential Equations, 218(2):444-515, 2005.

[23] A. Haro. Automatic differentiation tools in computational dynamical systems, 2008.

[24] C. Simó. On the analytical and numerical approximation of invariant manifolds. In Les Méthodes Modernes de la Mécanique Céleste. Modern Methods in Celestial Mechanics, volume 1, pages 285-329, 1990.

[25] A. Jorba and M. Zou. A software package for the numerical integration of ODEs by means of high-order Taylor methods. Experimental Mathematics, 14(1):99-117, 2005.

[26] O. Suvak and A. Demir. Quadratic approximations for the isochrons of oscillators: A general theory, advanced numerical methods and accurate phase computations. IEEE Transactions on Computer-Aided Design of Integrated Circuits and Systems, 29:1215-1228, 2010.

[27] M. H. Jensen, P. Bak, and T. Bohr. Transition to chaos by interaction of resonances in dissipative systems. I. Circle maps. Physical Review A, 30(4):1960, 1984.

[28] M. H. Jensen and S. Krishna. Inducing phase-locking and chaos in cellular oscillators by modulating the driving stimuli. FEBS letters, 586(11):1664-1668, 2012. 


\section{Appendix A: Computation of invariant curves using a Newton-like method}

In this section we develop the numerical scheme to compute the invariant curves of the dynamics of $F$. We will adapt the Newton-like method proposed in [21]. For the sake of self-containedness, we review the main steps of this method adapted to our problem. Since our problem is two-dimensional, there are significant simplifications compared to [21], where the method is presented in a setting of arbitrary dimension. However, if this method were to be applied to models of higher dimension, the structure would be basically the same.

Let $\varepsilon$ and $\omega$ be fixed. For the sake of simplicity, in this section we shall denote simply by $F$ the map $F_{\varepsilon, \omega}: \mathbb{T} \times \mathbb{R} \rightarrow \mathbb{T} \times \mathbb{R}$ defined in (10). Our main goal is to find a parameterization of an invariant curve, $\Gamma: \mathbb{T} \rightarrow \mathbb{T} \times \mathbb{R}$, of the map $F$. We note that in the special case $\varepsilon=0$, the limit cycle of the continuous system (1) in $(\theta, \sigma)$ coordinates is an invariant curve of the map (10). In this case, one has $\Gamma(\theta)=(\theta, 0)$. For $\varepsilon \neq 0$, an invariant curve can be done by solving an invariance equation of the following form:

$$
F(\Gamma(\theta))=\Gamma(f(\theta))
$$

where $\Gamma(\theta)$ (the parameterization of the curve) and $f(\theta)$ (the dynamics inside the curve) are unknowns. To perform the Newton-like method, we also consider the invariant normal (stable) bundle of $\Gamma(\theta)$, denoted by $N(\theta)$, and its linearized dynamics $\Lambda^{s}(\theta)$. The corresponding invariance equation to $N(\theta)$ and $\Lambda^{s}(\theta)$ is:

$$
D F(\Gamma(\theta)) N(\theta)=N(f(\theta)) \Lambda^{s}(\theta) .
$$

In the following we shall also denote $\Lambda(\theta)=\operatorname{diag}\left(\Lambda^{t}(\theta), \Lambda^{s}(\theta)\right)$ the linearized dynamics in both the tangent and normal bundle. Clearly, $\Lambda^{t}(\theta)=f^{\prime}(\theta)$.

At the $i$-th step of the method, we compute successive approximations $\Gamma_{i}(\theta), f_{i}(\theta), N_{i}(\theta)$ and $\Lambda_{i}(\theta)$ of $\Gamma(\theta), f(\theta), N(\theta)$ and $\Lambda(\theta)$, respectively, in two substeps. In the first substep we compute $\Gamma_{i}(\theta)$ and $f_{i}(\theta)$ and in the second substep we compute $N_{i}(\theta)$ and $\Lambda_{i}(\theta)$. Let us define $R_{i}(\theta)$ as the error in the invariance equation of the torus (24) at the step $i$ :

$$
R_{i}(\theta):=F\left(\Gamma_{i}(\theta)\right)-\Gamma_{i}\left(f_{i}(\theta)\right)
$$

Let $S_{i}^{s}(\theta)$ be the error in the invariance equation of the normal (stable) bundle (25) at the step $i$, that is:

$$
S_{i}^{s}(\theta):=D F\left(\Gamma_{i}(\theta)\right) N_{i}(\theta)-N_{i}\left(f_{i}(\theta)\right) \Lambda_{i}^{s}(\theta)
$$


We also define the adapted frame $P_{i}(\theta)=\left(D \Gamma_{i}(\theta), N_{i}(\theta)\right)$. Let $S_{i}(\theta)$ be the error of this adapted frame at the step $i$ :

$$
S_{i}(\theta):=D F\left(\Gamma_{i}(\theta)\right) P_{i}(\theta)-P_{i}(\theta) \Lambda_{i}(\theta) .
$$

One has that $S_{i}(\theta)=\left(D R_{i}(\theta), S_{i}^{s}(\theta)\right)$. In the following we denote $L_{i}(\theta):=D \Gamma_{i}(\theta)$.

In the first substep, we look for $\Gamma_{i+1}(\theta)$ and $f_{i+1}(\theta)$ of the following form:

$$
\begin{aligned}
\Gamma_{i+1}(\theta) & =\Gamma_{i}(\theta)+P_{i}(\theta) \xi_{i}(\theta) \\
f_{i+1}(\theta) & =f_{i}(\theta)+\varphi_{i}(\theta)
\end{aligned}
$$

where $\xi_{i}(\theta)$ and $\varphi_{i}(\theta)$ are the correction terms. To determine these correction terms, one proceeds as usual in Newton-like methods: first one substitutes expressions (26) and (27) in the invariance equation (24). Then one expands in Taylor series around $\Gamma_{i}(\theta)$ and $f_{i}(\theta)$ respectively, up to order two. Finally one imposes that all the terms up to order one in $\xi_{i}$ and $\varphi_{i}$ vanish, obtaining two equations for the unknowns $\xi_{i}$ and $\varphi_{i}$. Moreover, one can see that in this case we can take $\xi_{i}(\theta)$ of the form:

$$
\xi_{i}(\theta)=\left(\begin{array}{c}
0 \\
\xi_{i}^{s}(\theta)
\end{array}\right)
$$

so that we modify the invariant curve only in the normal (stable) direction. Following this procedure, one finds that $\xi_{i}^{s}(\theta)$ is the (unique) solution of

$$
\xi_{i}^{s}(\theta)=\Lambda_{i}^{s}\left(f_{i}^{-}(\theta)\right) \xi_{i}^{s}\left(f_{i}^{-}(\theta)\right)+\tilde{R}_{i}^{s}\left(f_{i}^{-}(\theta)\right)
$$

and $\varphi_{i}(\theta)$ as

$$
\varphi_{i}(\theta)=\tilde{R}_{i}^{t}(\theta)
$$

where $f_{i}^{-}(\theta)$ denotes an approximation of $f_{i}^{-1}(\theta)$, and

$$
\tilde{R}_{i}(\theta)=\left(\begin{array}{c}
\tilde{R}_{i}^{t}(\theta) \\
\tilde{R}_{i}^{s}(\theta)
\end{array}\right):=P_{i}^{-}\left(f_{i}(\theta)\right) R_{i}(\theta)
$$

being $P_{i}^{-}(\theta)$ an approximation of $P_{i}^{-1}(\theta)$. Elementary linear algebra shows that $\tilde{R}_{i}(\theta)$ is simply the error $R_{i}(\theta)$ in the basis $L_{i}\left(f_{i}(\theta)\right), N_{i}\left(f_{i}(\theta)\right)$.

Remark V.1 We point out that equation (28) is a fixed point equation of the form $\xi_{i}^{s}=\mathcal{F}\left(\xi_{i}^{s}, \theta\right)$. Moreover, $\mathcal{F}(\cdot, \theta)$ has Lipschitz constant $\Lambda_{i}^{s}\left(f_{i}^{-}(\theta)\right)<1$, so that equation (28) has a unique fixed point indeed. 
Moreover, one can solve this equation by iteration: first, one takes $\xi_{i, 0}^{s}(\theta)=\mathcal{F}(0, \theta)$. Then, for $j \geq 1$ one defines $\xi_{i, j}^{s}(\theta)=\mathcal{F}\left(\xi_{i, j-1}^{s}(\theta), \theta\right)$ and keeps iterating until the error $\left|\xi_{i, j}^{s}(\theta)-\mathcal{F}\left(\xi_{i, j}^{s}(\theta), \theta\right)\right|$ is sufficiently small.

In conclusion, after all these computations, $\Gamma_{i+1}(\theta)$ and $f_{i+1}(\theta)$ are defined as

$$
\begin{aligned}
\Gamma_{i+1}(\theta) & =\Gamma_{i}(\theta)+N_{i}(\theta) \xi_{i}^{s}(\theta), \\
f_{i+1}(\theta) & =f_{i}(\theta)+\tilde{R}_{i}^{t}(\theta) .
\end{aligned}
$$

We finish this substep by computing an approximation $f_{i+1}^{-}(\theta)$ of $f_{i+1}^{-1}(\theta)$, that will be used in the next step of the method. Let

$$
e_{i}(\theta)=f_{i}^{-}\left(f_{i+1}(\theta)\right)-\theta
$$

Then we define $f_{i+1}^{-}(\theta)$ as

$$
f_{i+1}^{-}(\theta)=f_{i}^{-}(\theta)-e_{i}\left(f_{i}^{-}(\theta)\right)
$$

This corresponds to one step of Newton's method for the equation

$$
f_{i+1}^{-} \circ f_{i+1}(\theta)-\theta=0 .
$$

In the second substep, we shall use $K_{i+1}(\theta), f_{i+1}(\theta)$ and $f_{i+1}^{-}(\theta)$ for the computation of $N_{i+1}(\theta)$ and $\Lambda_{i+1}^{s}(\theta)$. Again, we look for $N_{i+1}(\theta)$ and $\Lambda_{i+1}^{s}(\theta)$ of the following form:

$$
\begin{aligned}
& N_{i+1}(\theta)=N_{i}(\theta)+P_{i}(\theta) Q_{i}^{s}(\theta), \\
& \Lambda_{i+1}^{s}(\theta)=\Lambda_{i}^{s}(\theta)+\Delta_{i}^{s}(\theta),
\end{aligned}
$$

where $Q_{i}^{s}(\theta)$ and $\Delta_{i}(\theta)$ are the correction terms still to be determined. Analogously as in the previous substep, we substitute expressions (29) and (30) in the invariance equation (25), now taking of course $K_{i+1}(\theta)$ and $f_{i+1}(\theta)$. We note that equation (25) is linear with respect to $N(\theta)$ and $\Lambda^{s}(\theta)$, so that we can easily find equations for $Q_{i}^{s}(\theta)$ and $\Lambda_{i}^{s}(\theta)$ in order that (25) vanishes. Similarly as in the previous substep, one can choose $Q_{i}^{s}(\theta)$ of the form:

$$
Q_{i}^{s}(\theta)=\left(\begin{array}{c}
Q_{i}^{t s}(\theta) \\
0
\end{array}\right)
$$

that is, we correct the normal bundle in its complementary direction $L_{i}(\theta)$. Then one obtains that:

$$
Q_{i}^{t s}(\theta)=\left(Q_{i}^{t s}\left(f_{i+1}^{-}(\theta)\right) \Lambda_{i}^{s}(\theta)-\tilde{S}_{i}^{t s}(\theta)\right)\left(\Lambda_{i}^{t}(\theta)\right)^{-1},
$$


and:

$$
\Delta_{i}^{s}(\theta)=\tilde{S}_{i}^{s s}(\theta)
$$

where:

$$
\tilde{S}_{i}^{s}(\theta)=\left(\begin{array}{c}
\tilde{S}_{i}^{t s}(\theta) \\
\tilde{S}_{i}^{s s}(\theta)
\end{array}\right):=P_{i}^{-}\left(f_{i+1}(\theta)\right) S_{i}^{s}(\theta)
$$

We point out that, analogously as $\tilde{P}_{i}(\theta), \tilde{S}_{i}^{s}(\theta)$ is the error of the normal bundle $S_{i}^{s}(\theta)$ in the basis $L_{i}\left(f_{i}(\theta)\right)$, $N_{i}\left(f_{i}(\theta)\right)$. Again, equation (31) can be solved with the procedure described in Remark V.1. After that, we define $N_{i+1}(\theta)$ and $\Lambda_{i+1}(\theta)$ as:

$$
\begin{aligned}
& N_{i+1}(\theta)=N_{i}(\theta)+L_{i}(\theta) Q_{i}^{t s}(\theta), \\
& \Lambda_{i+1}^{s}(\theta)=\Lambda_{i}^{s}(\theta)+\tilde{S}_{i+1}^{s s}(\theta), \\
& \Lambda_{i+1}^{t}(\theta)=f_{i+1}^{\prime}(\theta) .
\end{aligned}
$$

To finish, we compute the approximation $P_{i+1}^{-}(\theta)$ of $P_{i+1}^{-1}(\theta)$ which shall be used in the next iteration of the method. Let:

$$
E_{i}(\theta)=P_{i}^{-}(\theta) P_{i+1}(\theta)-\text { Id }
$$

Then we define $P_{i+1}^{-}(\theta)$ as:

$$
P_{i+1}^{-}(\theta)=P_{i}^{-}(\theta)-E_{i}(\theta) P_{i}(\theta)
$$

Again, this corresponds to one step of Newton's method for the equation:

$$
P_{i+1}^{-}(\theta) P_{i+1}(\theta)-\mathrm{Id}=0
$$

\section{A. Choosing the initial seeds}

In this subsection we indicate how to choose initial seeds for the Newton method, as proposed in [21]. We point out that we are in a perturbative setting, that is the map $F$ depends on a parameter $\varepsilon$, so that one can take advantage of it.

Indeed, for an initial value $\varepsilon=\varepsilon_{0}$, that we assume to be sufficiently small, we can take the initial seeds $\Gamma_{0}(\theta), f_{0}(\theta), N_{0}(\theta)$ and $\Lambda_{0}(\theta)$ (and also $P_{0}(\theta), P_{0}^{-}(\theta)$ and $f_{0}^{-}(\theta)$ ) simply as the corresponding objects for 
$\varepsilon=0$. In our setting, one has:

$$
\begin{aligned}
& \Gamma_{0}(\theta)=\left(\begin{array}{l}
\theta \\
0
\end{array}\right), \\
& f_{0}(\theta)=\theta+\omega, \quad f_{0}^{-}(\theta)=\theta-\omega \\
& L_{0}(\theta)=\left(\begin{array}{l}
1 \\
0
\end{array}\right), \quad N_{0}(\theta)=\left(\begin{array}{l}
0 \\
1
\end{array}\right), \\
& P_{0}(\theta)=P_{0}^{-}(\theta)=\left(\begin{array}{ll}
1 & 0 \\
0 & 1
\end{array}\right), \\
& \Lambda_{0}(\theta)=\left(\begin{array}{cc}
\Lambda_{0}^{t}(\theta) & 0 \\
0 & \Lambda_{0}^{s}(\theta)
\end{array}\right)=\left(\begin{array}{ll}
1 & 0 \\
0 & e^{\lambda \omega}
\end{array}\right) .
\end{aligned}
$$

For $\varepsilon>\varepsilon_{0}$, one can perform a continuation method to find good initial seeds for successive values of $\varepsilon$. In [21], the authors propose to perform a continuation method just for the parameterization of the torus, $\Gamma(\theta)$, and its internal dynamics $f(\theta)$, and omit the normal bundle $N(\theta)$ and the linearized dynamics $\Lambda(\theta)$. We now describe this continuation method.

Assume that for a given $\varepsilon$ we have good approximations $\Gamma^{\varepsilon}(\theta)$ and $f^{\varepsilon}(\theta)$ of $\Gamma(\theta), f(\theta)$ respectively. Then, we define the initial seeds of the Newton method for the parameter $\varepsilon+h$ as:

$$
\begin{aligned}
\Gamma_{0}^{\varepsilon+h}(\theta) & =\Gamma^{\varepsilon}(\theta)+\frac{\partial \Gamma^{\varepsilon}}{\partial \varepsilon}(\theta) h, \\
f_{0}^{\varepsilon+h}(\theta) & =f^{\varepsilon}(\theta)+\frac{\partial f^{\varepsilon}}{\partial \varepsilon}(\theta) h .
\end{aligned}
$$

One can obtain the following invariance equation for $\frac{\partial \Gamma^{\varepsilon}}{\partial \varepsilon}$ and $\frac{\partial f^{\varepsilon}}{\partial \varepsilon}$ just by differentiating (24) with respect to $\varepsilon$ :

$$
D F\left(\Gamma^{\varepsilon}(\theta)\right) \frac{\partial \Gamma^{\varepsilon}}{\partial \varepsilon}(\theta)=D \Gamma^{\varepsilon}\left(f^{\varepsilon}(\theta)\right) \frac{\partial f^{\varepsilon}}{\partial \varepsilon}(\theta)+\frac{\partial \Gamma^{\varepsilon}}{\partial \varepsilon}(\theta) .
$$

Now, writing $\frac{\partial \Gamma^{\varepsilon}}{\partial \varepsilon}$ and $\frac{\partial f^{\varepsilon}}{\partial \varepsilon}$ in the basis $L^{\varepsilon}(\theta), N^{\varepsilon}(\theta)$ we obtain:

$$
\begin{gathered}
\frac{\partial \Gamma^{\varepsilon}}{\partial \varepsilon}(\theta)=P^{\varepsilon}(\theta) \xi^{\varepsilon}(\theta), \\
\frac{\partial f^{\varepsilon}}{\partial \varepsilon}(\theta)=\varphi^{\varepsilon}(\theta),
\end{gathered}
$$

for some unknowns $\xi^{\varepsilon}(\theta)$ and $\varphi^{\varepsilon}(\theta)$. Again, $\xi^{\varepsilon}(\theta)$ can be taken of the form:

$$
\xi^{\varepsilon}(\theta)=\left(\begin{array}{c}
0 \\
\xi^{s, \varepsilon}(\theta)
\end{array}\right),
$$


so that we just correct the torus in the normal direction. Let:

$$
R^{\varepsilon}(\theta)=\frac{\partial F}{\partial \varepsilon}\left(\Gamma^{\varepsilon}(\theta)\right), \quad \tilde{R}^{\varepsilon}(\theta)=\left(P^{\varepsilon}\left(f^{\varepsilon}(\theta)\right)\right)^{-1} R^{\varepsilon}(\theta) .
$$

Then, performing the standard computations of Newton's method, one obtains the following identities for $\xi^{s, \varepsilon}$ and $\varphi^{\varepsilon}$ :

$$
\begin{aligned}
\xi^{s, \varepsilon}(\theta) & =\Lambda^{s, \varepsilon}\left(f_{i}^{-, \varepsilon}(\theta)\right) \xi^{s, \varepsilon}\left(f_{i}^{-, \varepsilon}(\theta)\right)+\tilde{R}^{s, \varepsilon}\left(f_{i}^{-, \varepsilon}(\theta)\right), \\
\varphi^{\varepsilon}(\theta) & =\tilde{R}^{t, \varepsilon}(\theta) .
\end{aligned}
$$

As above, the equation for $\xi^{s, \varepsilon}$ has a unique solution that can be found with the method described in Remark V.1. In conclusion, after finding the corrections, we take the initial seeds $\Gamma_{0}^{\varepsilon+h}(\theta)$ and $f_{0}^{\varepsilon+h}(\theta)$ as:

$$
\begin{gathered}
\Gamma_{0}^{\varepsilon+h}(\theta)=\Gamma^{\varepsilon}(\theta)+N^{\varepsilon}(\theta) \xi^{s, \varepsilon}(\theta) h, \\
f_{0}^{\varepsilon+h}(\theta)=f^{\varepsilon}(\theta)+\tilde{R}^{t, \varepsilon}(\theta) h,
\end{gathered}
$$

and then we proceed again with the Newton-like method described above. 


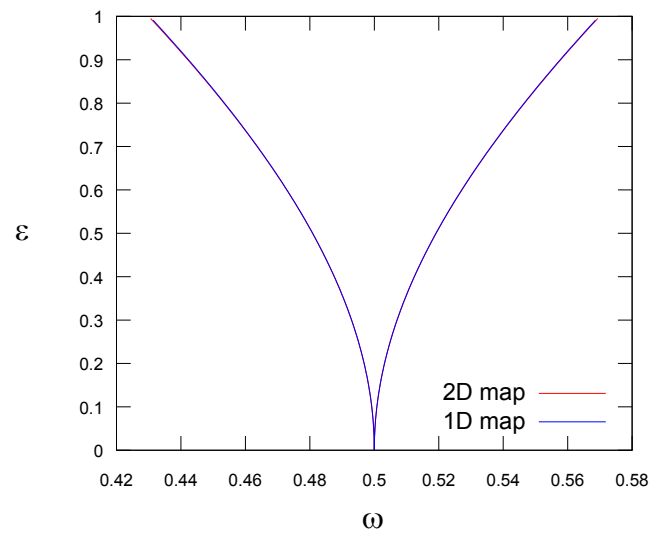

(a) $p / q=1 / 2$

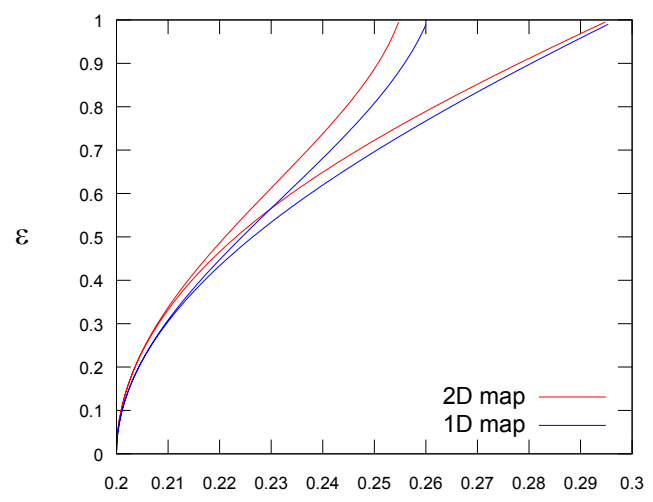

$\omega$

(c) $p / q=1 / 5$

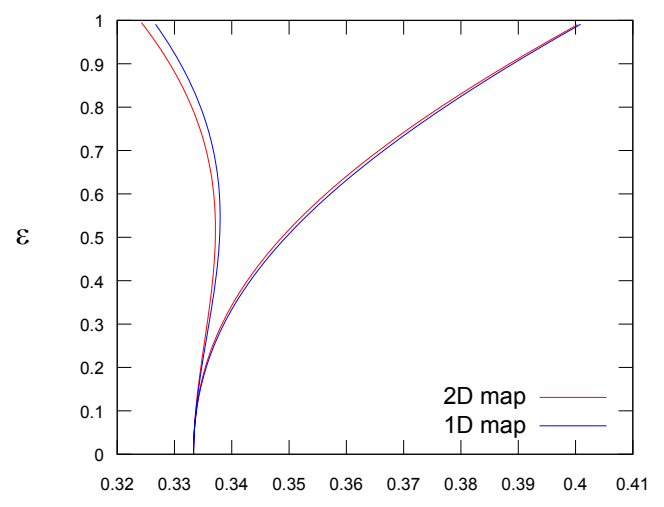

$\omega$

(b) $p / q=1 / 3$

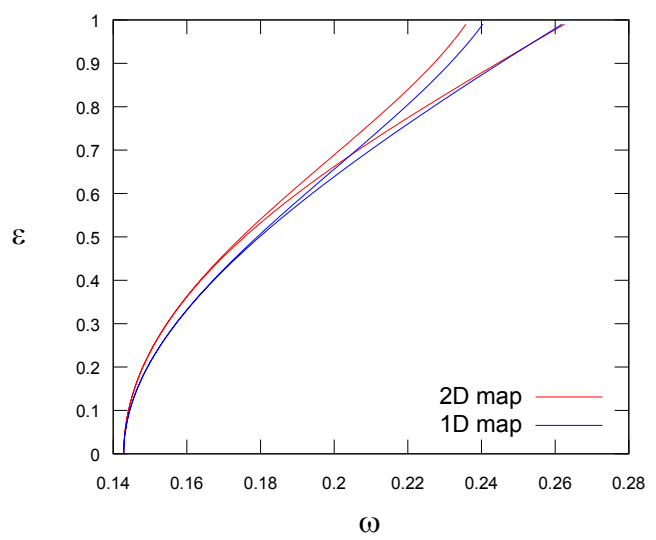

(d) $p / q=1 / 7$

Figure 9: Comparison between different $p / q$-Arnold tongues of the 2D (red) and the 1D (blue) maps,

with $\alpha=5, a=1$. Observe how the tangency between the two boundary gets more pronounced when increasing the denominator $q$. It can be prove that the tangency is of order $q$. 


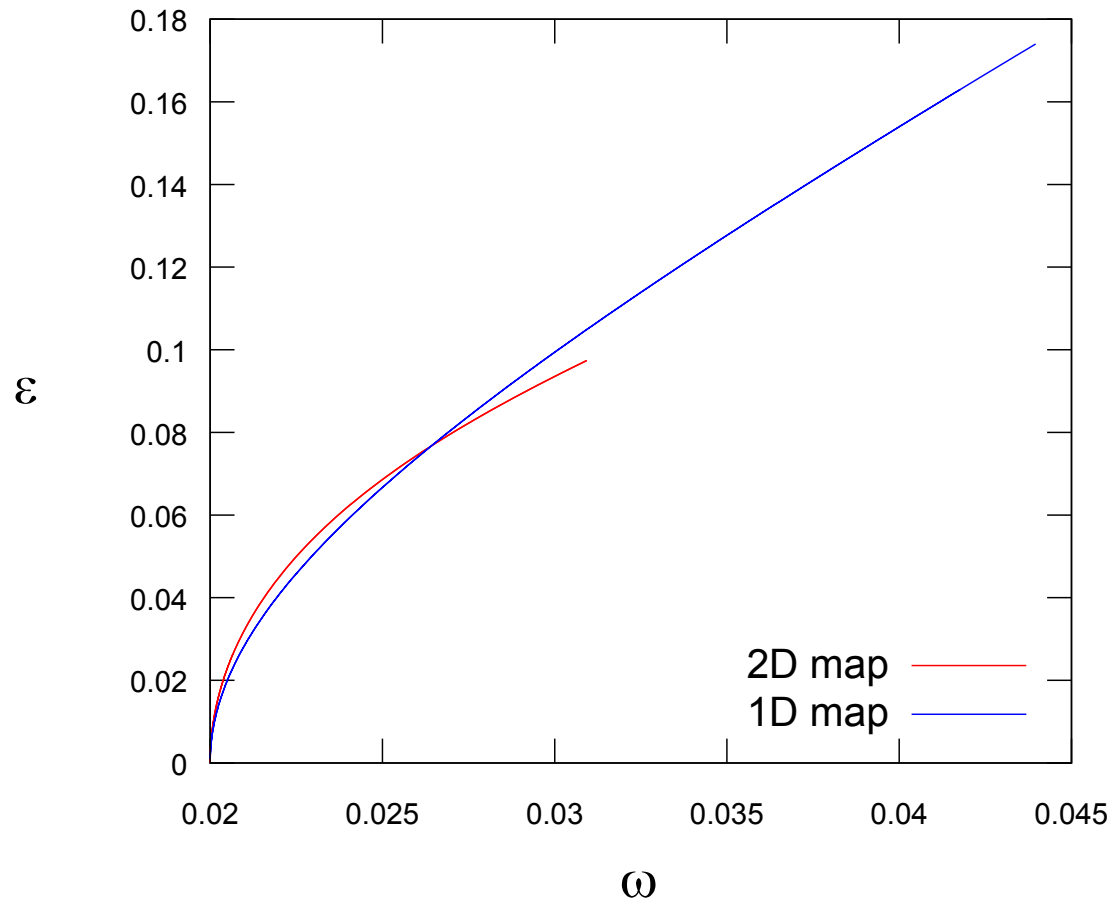

Figure 10: Comparison between one of the boundaries of the 1/50-Arnold tongue of the 2D and the $1 D$ maps, with $\alpha=5, a=1$. 

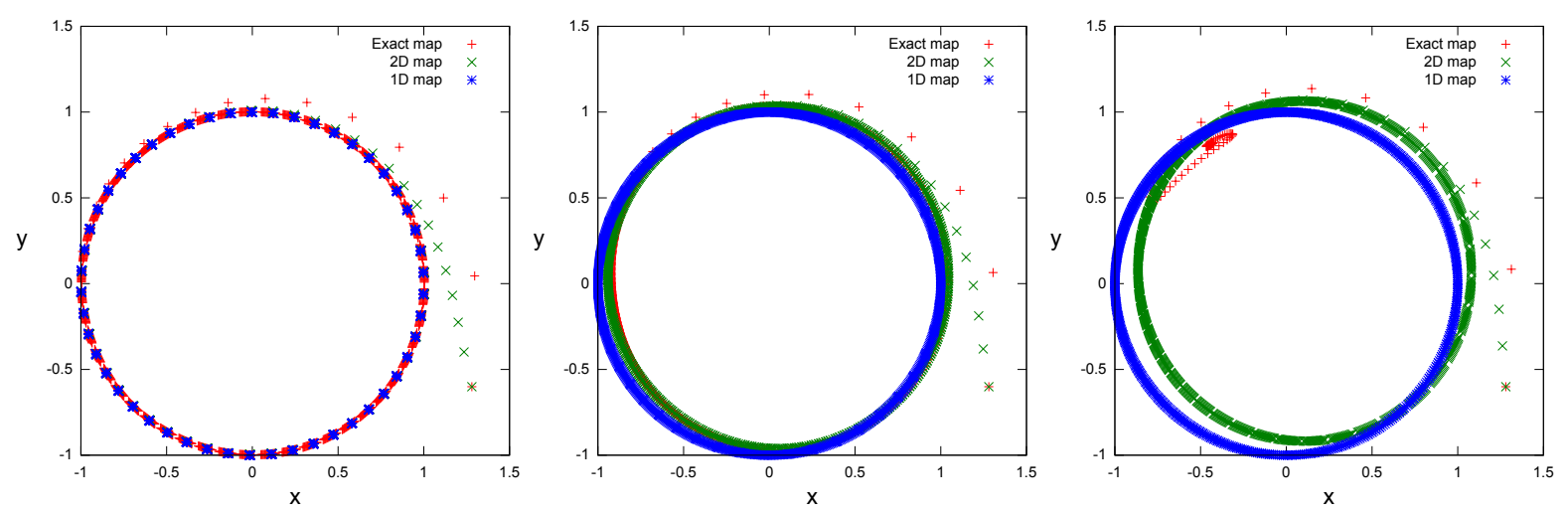

(a) $\varepsilon=0.001$.

(b) $\varepsilon=0.017$.

(c) $\varepsilon=0.033$.
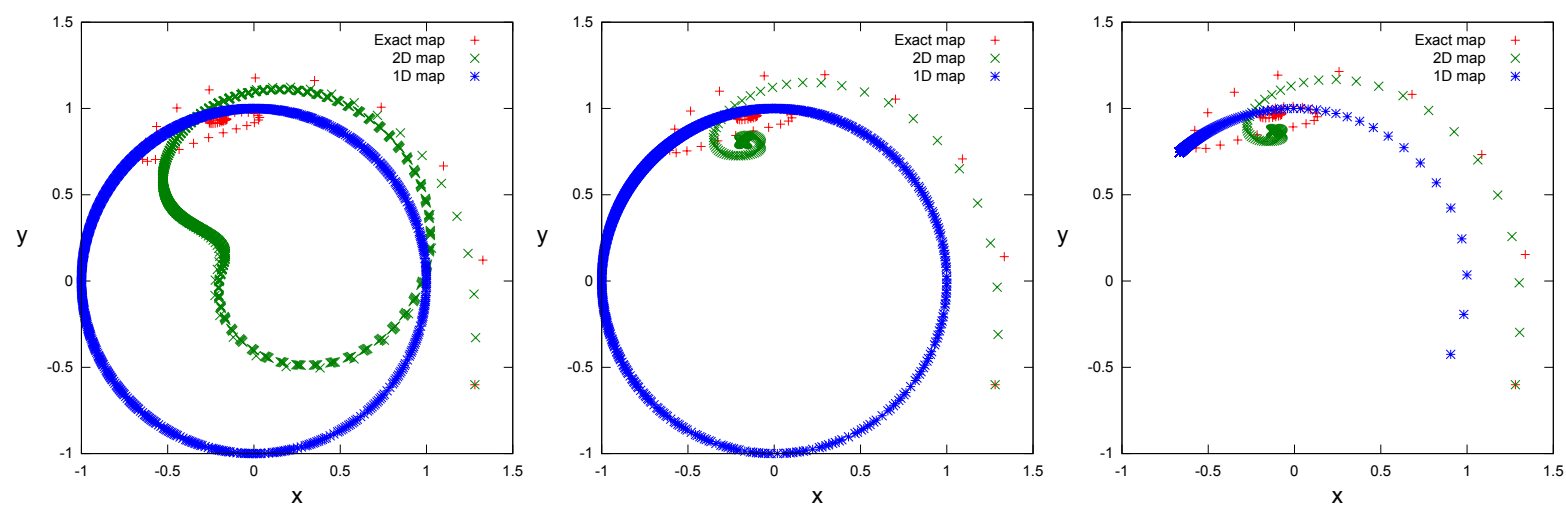

(d) $\varepsilon=0.063$.

(e) $\varepsilon=0.079$.

(f) $\varepsilon=0.089$.

Figure 11: Evolution of the asymptotic states of the exact (red), 2D (8) (green) and 1D maps (9) (blue). Simulations for $\alpha=5, a=1, \omega=1 / 50$ and different values of $\varepsilon$. 

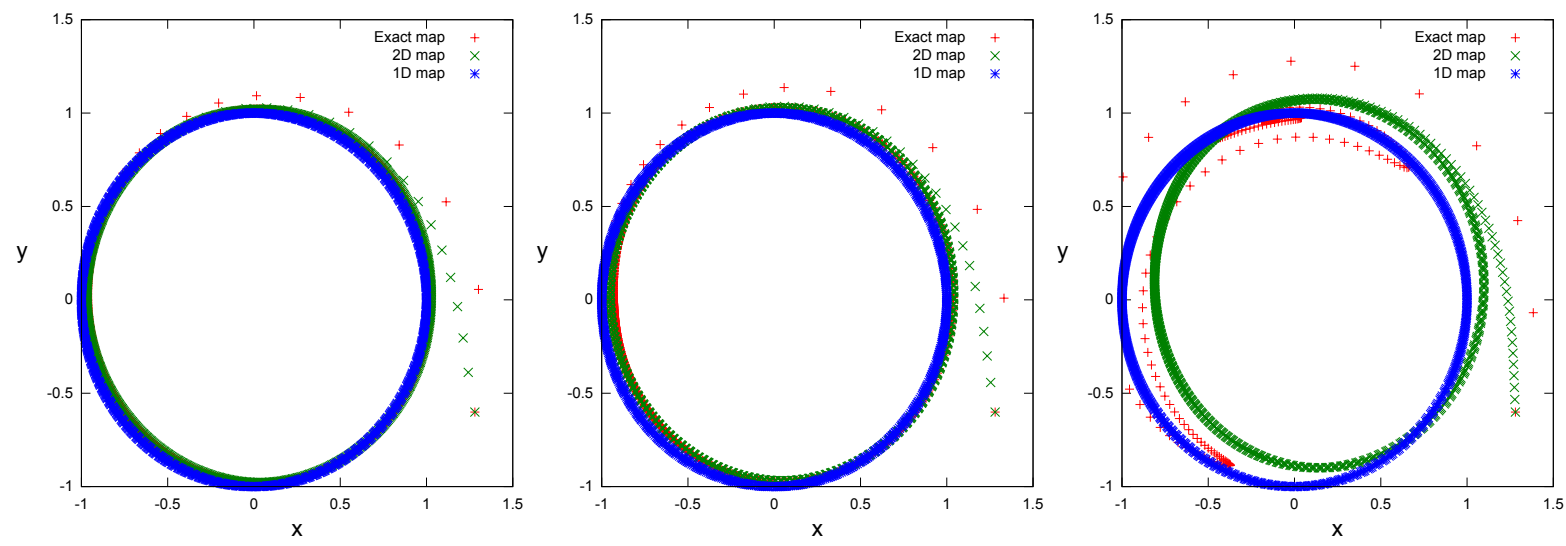

(a) $\omega=1 / 50$

(b) $\omega=1 / 70$.

(c) $\omega=1 / 190$.
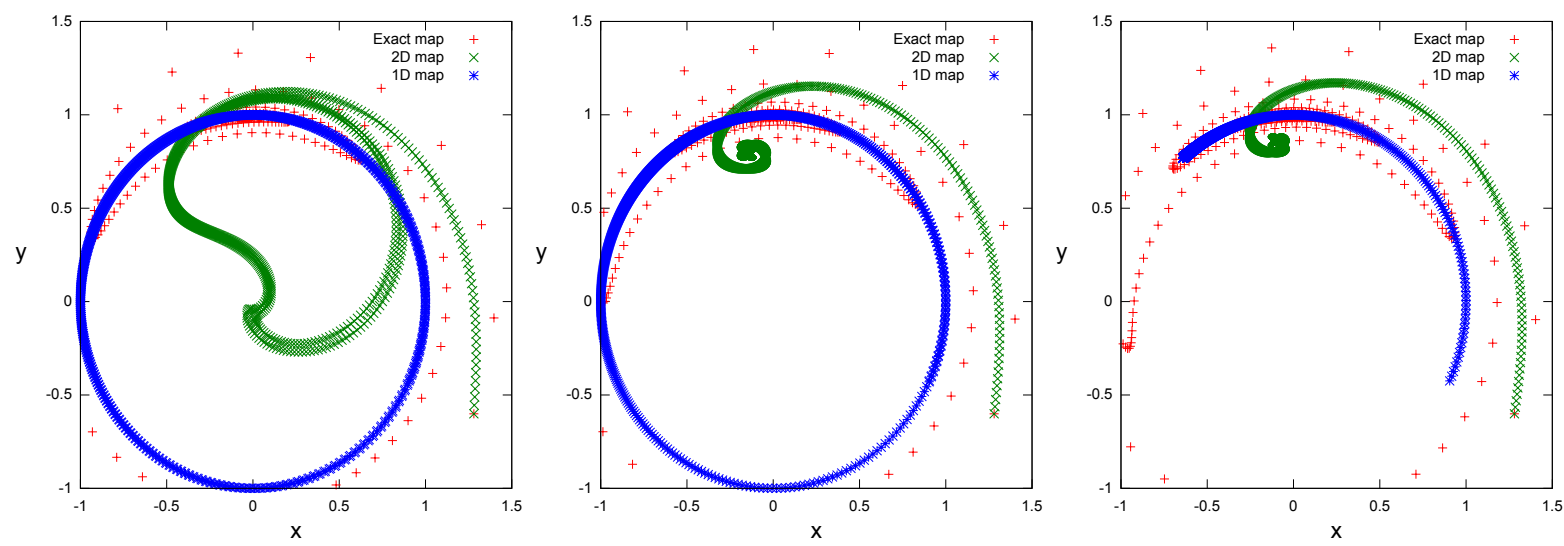

(d) $\omega=1 / 310$.

(e) $\omega=1 / 390$.

(f) $\omega=1 / 440$.

Figure 12: Evolution of the asymptotic states of the exact (red), 2D (8) (green) and 1D maps (9) (blue). Simulations for $\alpha=5, a=1, \varepsilon=0.01$ and different values of $\omega$. 


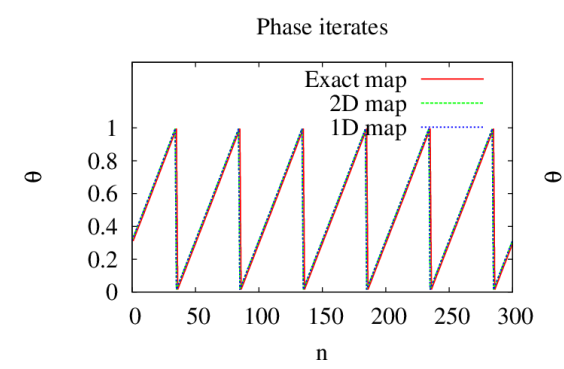

(a) $\varepsilon=0.001$.

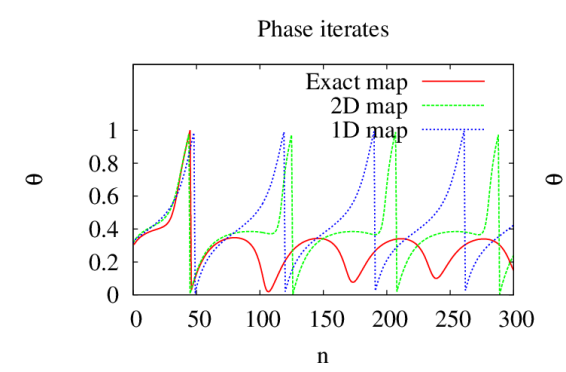

(d) $\varepsilon=0.063$.

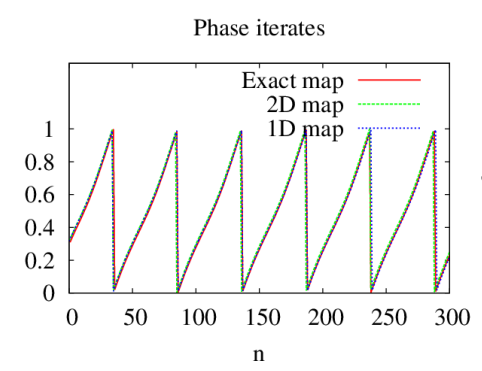

(b) $\varepsilon=0.017$.

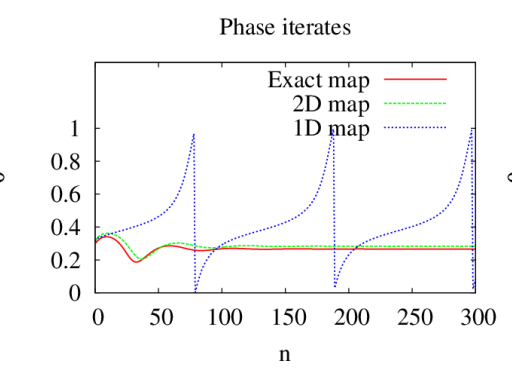

(e) $\varepsilon=0.079$.

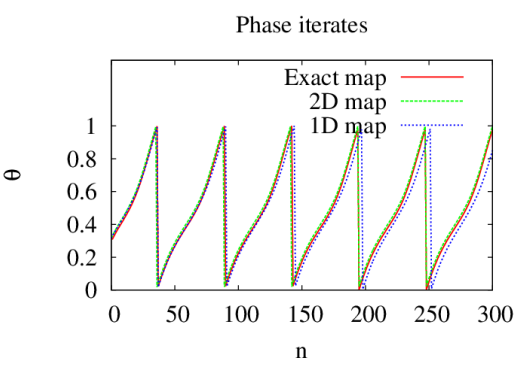

(c) $\varepsilon=0.033$.

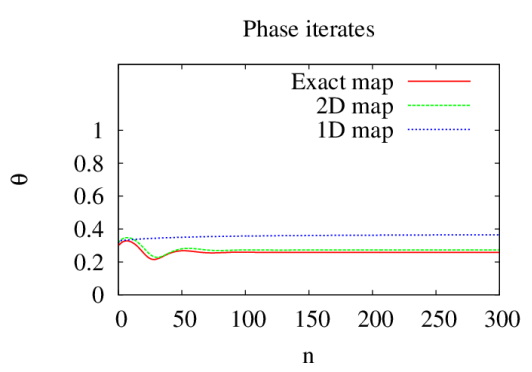

(f) $\varepsilon=0.089$.

Figure 13: Evolution of the phase variable for the exact (red), 2D maps (8) (green) and 1D (9) (blue).

Simulations for $\alpha=5, a=1, \omega=1 / 50$ and different values of $\varepsilon$. 